\title{
THE DISCONTINUOUS INITIAL VALUE PROBLEM OF A REACTING GAS FLOW SYSTEM
}

BY

\author{
YING LUNG-AN AND WANG CHING-HUA
}

\begin{abstract}
We show that the local solvability of the solution of a reacting gas flow system (1.1)-(1.4) with initial values (1.5), which has a large jump at the point $x^{\prime}=0$ and the structure of the solution near the origin $(0,0)$ are identical to those of the Riemann problem of the homogeneous system corresponding to the Cauchy problem (1.1)-(1.5).
\end{abstract}

1. Introduction. If we neglect the effects of diffusion, friction and heat conduction within a fluid containing two species with a chemical reaction, the system of equations governing this fluid can be written as

$$
\begin{gathered}
\partial \rho_{1} / \partial t+\partial\left(\rho_{1} u\right) / \partial x^{\prime}=g\left(\rho_{1}, \rho_{2}, T\right) \quad \text { (conservation of mass), } \\
\partial \rho_{2} / \partial t+\partial\left(\rho_{2} u\right) / \partial x^{\prime}=-g\left(\rho_{1}, \rho_{2}, T\right) \quad \\
\partial(\rho u) / \partial t+\partial\left(\rho u^{2}+p\right) / \partial x^{\prime}=0 \quad \text { (conservation of momentum), } \\
(\partial / \partial t)\left[\rho_{1}\left(e_{1}+u^{2} / 2\right)+\rho_{2}\left(e_{2}+u^{2} / 2\right)\right] \\
+\left(\partial / \partial x^{\prime}\right)\left[\rho_{1} u\left(u^{2} / 2+e_{1}+p_{1} v_{1}\right)+\rho_{2} u\left(u^{2} / 2+e_{2}+p_{2} v_{2}\right)\right]=0
\end{gathered}
$$

(conservation of energy),

where $t \geqslant 0, x^{\prime} \in(-\infty,+\infty)$ denote the Euler coordinates of time and space, $u$ denotes the velocity, $\rho_{i}, v_{i}, p_{i}, e_{i}(i=1,2), T$ denote the state parameters: density, specific volume, pressure, energy per unit volume and temperature, respectively; $v_{i}=1 / \rho_{i} ; p=p_{1}+p_{2}$ and $\rho=\rho_{1}+\rho_{2}$ are called the total pressure and total density. Functions $g$ and $-g$ are the rate of chemical reaction, which depends on temperature and the density of these two species. Here, for simplification, we only consider two reacting materials, but our result can be extended to any number of reacting materials without any difficulty; the sum of the rates of chemical reaction equals zero because of the conservation of total mass. For the concrete form of the function $g\left(\rho_{1}, \rho_{2}, T\right)$ and the general system, including the effects of diffusion, friction and heat conduction, see [1].

In the following, we introduce, for convenience, another state parameter, entropy, $S_{i}(i=1,2)$. There are some state equations connecting all of the state parameters. We assume both materials are ideal gases, i.e.,

$$
p_{1} v_{1}=p_{2} v_{2}=R T
$$

Received by the editors January 31, 1980 and, in revised form, April 14, 1980. 1980 Mathematics Subject Classification. Primary 35L60. 
where $R$ is the gas constant. We also assume they are polytropic, i.e.,

$$
p_{i}=a^{2} \exp \left\{\left(\gamma_{i}-1\right) s_{i} / R\right\} v_{i}^{-\gamma_{i}} \quad(i=1,2)
$$

where $a^{2}$ is a positive constant, $1<\gamma_{i}<\frac{5}{3}$. In addition, if we consider the state equation

$$
e_{i}=p_{i} v_{i} /\left(\gamma_{i}-1\right)+q_{i}, \quad q_{i}=\text { constant }(i=1,2),
$$

then the system of (1.1)-(1.4) takes a closed form. We can eliminate some of the unknowns in (1.1) and get a system for four unknowns, for instance, $\rho_{1}, \rho_{2}, u$ and $p$.

(1.1)-(1.4) is a nonhomogeneous nonlinear hyperbolic system, the classical solutions of which have been quite sufficiently investigated by many authors [2]-[8]. Wang Rou-Hwai and Wuu Jwo-Chun investigated the classical solutions of the mixed boundary value problems [9].

The problem of solvability and the structure of discontinuous solutions are of both theoretical and practical interest. If the initial values have the first kind of discontinuity at the origin and are smooth elsewhere, Gu Chao-Hao, Lee Da-Tsin, Ho Zon-Yi [10]-[12], and Roždestvenskii and Yanenko [13] investigated the Cauchy problem in the case $n=2$; they proved that the local solutions exist and the structure of the solutions is identical with that of the Riemann problem. Lee $\mathrm{Da}-\mathrm{Tsin}$ and $\mathrm{Yu}$ Wen-Tzu investigated the general case when $n \geqslant 2$; they assumed that the jump at the origin is sufficiently small [14]-[17].

It is the purpose of this paper to discuss the local solvability and the local structure of the solution of the system (1.1)-(1.4) with initial values

$$
\left(\rho_{1}\left(x^{\prime}, 0\right), \rho_{2}\left(x^{\prime}, 0\right), u\left(x^{\prime}, 0\right), p\left(x^{\prime}, 0\right)\right)=\left(\rho_{1,0}\left(x^{\prime}\right), \rho_{2,0}\left(x^{\prime}\right), u_{0}\left(x^{\prime}\right), p_{0}\left(x^{\prime}\right)\right),
$$

where the functions $\rho_{10}, \rho_{20}, u_{0}, p_{0}$ have a large jump at the point $x^{\prime}=0$. We will prove that the Cauchy problem (1.1)-(1.5) is locally solvable and the structure of the solution near the point $(0,0)$ is identical to that of the homogeneous system corresponding to (1.1).

$$
\begin{aligned}
& \partial \rho_{1} / \partial t+\partial\left(\rho_{1} u\right) / \partial x^{\prime}=0, \quad \partial \rho_{2} / \partial t+\partial\left(\rho_{2} u\right) / \partial x^{\prime}=0 \\
& \partial(\rho u) / \partial t+\partial\left(\rho u^{2}+p\right) / \partial x^{\prime}=0 \\
& (\partial / \partial t)\left[\rho_{1}\left(e_{1}+u^{2} / 2\right)+\rho_{2}\left(e_{2}+u^{2} / 2\right)\right] \\
& \quad+\left(\partial / \partial x^{\prime}\right)\left[\rho_{1} u\left(u^{2} / 2+e_{1}+p_{1} v_{1}\right)+\rho_{2} u\left(u^{2} / 2+e_{2}+p_{2} v_{2}\right)\right]=0
\end{aligned}
$$

with the initial values

$$
\left(\rho_{1}\left(x^{\prime}, 0\right), \rho_{2}\left(x^{\prime}, 0\right), u\left(x^{\prime}, 0\right), p\left(x^{\prime}, 0\right)\right)= \begin{cases}\left(\rho_{1 l}, \rho_{2 l}, u_{l}, p_{l}\right), & x^{\prime}<0 \\ \left(\rho_{1 r}, \rho_{2 r}, u_{r}, p_{r}\right), & x^{\prime}>0 .\end{cases}
$$

In $\S \S 2$ and 3 we give some expressions of this system, its characteristic and jump relations, some properties of the jump relations and a description of the Riemann problem, all of which will be useful later. In $\$ 4$, we prove the existence of a boundary value problem, the solution of which is called the rarefaction wave; this result has been obtained for $n=2$ [13]. There is an essential difference between the cases $n>2$ and $n=2$. We prove this result for $n=4$, but our method can be used for any $n$ without difficulty. In $\$ 5$ we prove: the local existence and local structure 
theorem when there is a rarefaction wave and a shock wave; the local existence of a mixed boundary value problem where the solution has a contact discontinuity; and the existence of a shock wave using the Schauder Fixed Point Theorem. In §6, we give a brief discussion of the other cases.

We are very grateful to Professors P. D. Lax and Gu Chao-Hao for helpful discussions.

2. The characteristic and jump relations. In the following, we always discuss system (1.1) under the Lagrange coordinate system. Set

$$
x=\int_{a(t)}^{x^{\prime}} \rho(\xi, t) d \xi
$$

where $a(t)$ is the space coordinate of the material point starting form $(0,0)$; then (1.1) can be written in the equivalent form

$$
\begin{aligned}
& \partial v / \partial t-\partial u / \partial x=0, \quad \partial c / \partial t=f(v, p, c), \quad \partial u / \partial t+\partial p / \partial x=0 \\
& \frac{\partial}{\partial t}\left(\frac{p v}{\gamma_{c}-1}+\frac{u^{2}}{2}+c q_{1}+(1-c) q_{2}\right)+\frac{\partial}{\partial x}(p u)=0
\end{aligned}
$$

where $v=v_{1} v_{2} /\left(v_{1}+v_{2}\right), c=v / v_{1}, f=g v$, and

$$
1 /\left(\gamma_{c}-1\right)=c /\left(\gamma_{1}-1\right)+(1-c) /\left(\gamma_{2}-1\right) \text {. }
$$

(2.1) is a first order, quasilinear, hyperbolic system satisfied by the unknowns $u, p$, $c, v$. Let

$$
s=R\left(\gamma_{c}-1\right)^{-1} \log a^{-2} v_{c p} \equiv s(v, p, c),
$$

which is called total entropy. (2.1) can be written as

$$
\frac{\partial}{\partial t}\left(\begin{array}{l}
u \\
p \\
c \\
s
\end{array}\right)+\left(\begin{array}{cccc}
0 & 1 & 0 & 0 \\
-\left(v_{p}\right)^{-1} & 0 & 0 & 0 \\
0 & 0 & 0 & 0 \\
0 & 0 & 0 & 0
\end{array}\right) \frac{\partial}{\partial x}\left(\begin{array}{l}
u \\
p \\
c \\
s
\end{array}\right)=\left(\begin{array}{l}
0 \\
0 \\
f \\
h
\end{array}\right)
$$

where $v_{p}=(\partial v / \partial p)_{s, c}$, which is expressed as the partial derivative looking upon $v$ as the function of $p, s, c$ in (2.3), and

$$
h=\frac{\left(\gamma_{1}-\gamma_{2}\right)\left(\gamma_{c}-1\right)}{\left(\gamma_{1}-1\right)\left(\gamma_{2}-1\right)}\left(-\frac{s}{\gamma_{c}}+\frac{R}{\gamma_{c}} \log \left(a^{2} p^{-1}\right)+\frac{R}{\gamma_{c}-1}\right) f-R\left(q_{1}-q_{2}\right) f / p v .
$$

Let

$$
w=\left(\begin{array}{l}
u \\
p \\
c \\
s
\end{array}\right), \quad A(w)=\left(\begin{array}{cccc}
0 & 1 & 0 & 0 \\
-\left(v_{p}\right)^{-1} & 0 & 0 & 0 \\
0 & 0 & 0 & 0 \\
0 & 0 & 0 & 0
\end{array}\right), \quad F(w)=\left(\begin{array}{l}
0 \\
0 \\
f \\
h
\end{array}\right)
$$

Then (2.4) can be written as

$$
\partial w / \partial t+A(w)(\partial w / \partial x)=F(w) .
$$


Let $\lambda_{i}(w), \bar{l}_{i}(w)(i=1,2,3,4)$ be the eigenvalues and left eigenvectors of the matrix $A(w)$; then left multiply $(2.5)$ by $\bar{l}_{i}(w)$ to obtain

$$
\bar{l}_{i}(w)\left(d w / d t_{i}\right)=b_{i}(w) \quad(i=1,2,3,4)
$$

where

$$
\frac{d}{d t_{i}}=\frac{\partial}{\partial t}+\lambda_{i}(w) \frac{\partial}{\partial x}, \quad b_{i}(w)=\bar{l}_{i}(w) F(w)
$$

We have

$$
\begin{array}{lll}
\lambda_{1}=-1 / \sqrt{-v_{p}}, & \lambda_{2}=\lambda_{3}=0, & \lambda_{4}=1 / \sqrt{-v_{p}} . \\
i_{1}=\left(1,-\sqrt{-v_{p}}, 0,0\right), & \bar{l}_{2}=(0,0,1,0), & \\
\bar{l}_{3}=(0,0,0,1), & \bar{l}_{4}=\left(1, \sqrt{-v_{p}}, 0,0\right) .
\end{array}
$$

Hence (2.6) is

$$
\begin{aligned}
& d u / d t_{1}+l_{12}(p, c, s)\left(d p / d t_{1}\right)=0, \quad \partial c / \partial t=f \\
& \partial s / \partial t=h, \quad d u / d t_{4}+l_{42}(p, c, s)\left(d p / d t_{4}\right)=0
\end{aligned}
$$

where

$$
l_{12}(p, c, s)=-l_{42}(p, c, s)=-\sqrt{-v_{p}} .
$$

We define

$$
r_{1}=u-\int_{1}^{p} \sqrt{-v_{p}} d p, \quad r_{2}=c, \quad r_{3}=s, \quad r_{4}=u+\int_{1}^{p} \sqrt{-v_{p}} d p
$$

Then (2.7) can be written as

$$
\left[\begin{array}{cccc}
\frac{d}{d t_{1}} & \int_{1}^{p} \frac{\partial \sqrt{-v_{p}}}{\partial c} d p \frac{d}{d t_{1}} & \int_{1}^{p} \frac{\partial \sqrt{-v_{p}}}{\partial s} d p \frac{d}{d t_{1}} & 0 \\
0 & \frac{\partial}{\partial t} & 0 & 0 \\
0 & 0 & \frac{\partial}{\partial t} & 0 \\
0 & -\int_{1}^{p} \frac{\partial \sqrt{-v_{p}}}{\partial c} d p \frac{d}{d t_{4}} & -\int_{1}^{p} \frac{\partial \sqrt{-v_{p}}}{\partial s} d p \frac{d}{d t_{4}} & \frac{d}{d t_{4}}
\end{array}\right]\left(\begin{array}{l}
r_{1} \\
r_{2} \\
r_{3} \\
r_{4}
\end{array}\right)=\left(\begin{array}{l}
0 \\
f \\
h \\
0
\end{array}\right)
$$

Let

$$
r=\left[\begin{array}{l}
r_{1} \\
r_{2} \\
r_{3} \\
r_{4}
\end{array}\right], \quad \begin{aligned}
& l_{1}(r)=\left(1, \int_{1}^{p} \frac{\partial \sqrt{-v_{p}}}{\partial c} d p, \int_{1}^{p} \frac{\partial \sqrt{-v_{p}}}{\partial s} d p, 0\right), \\
& l_{3}(r)=(0,1,0,0), \\
& l_{4}(r)=\left(1,-\int_{1}^{p} \frac{\partial \sqrt{-v_{p}}}{\partial c} d p,-\int_{1}^{p} \frac{\partial \sqrt{-v_{p}}}{\partial s} d p, 1\right) .
\end{aligned}
$$


Then (2.7) can be written in the equivalent form

$$
l_{i}(r)\left(d r / d t_{i}\right)=b_{i}(r) \quad(i=1,2,3,4),
$$

where $b_{i}$ denotes the right-side functions as before, but it should be noted that the independent variable is $r$. The difference between (2.12) and (2.7) is that the fourth components of the left eigenvectors $l_{i}(i=1,2,3)$ vanish in (2.12).

We have derived the different forms of reacting gas flow equations which are equivalent to each other; we will use them when convenient.

Now we discuss jump relations; from (2.1) it is easy to obtain

$$
\begin{gathered}
\left(u-u_{r}\right) /\left(p-p_{r}\right)=\sqrt{2 v_{r} /\left[\left(\gamma_{c, r}+1\right) p+\left(\gamma_{c, r}-1\right) p_{r}\right]}, \\
c=c_{r}, \\
v / v_{r}=\left[\left(\gamma_{c, r}-1\right) p+\left(\gamma_{c, r}+1\right) p_{r}\right] /\left[\left(\gamma_{c, r}+1\right) p+\left(\gamma_{c, r}-1\right) p_{r}\right],
\end{gathered}
$$

where the subscript $r$ denotes the right limit (for $x$ ) of the solution at a discontinuous point, the quantity without subscript denotes the left limit, $\gamma_{c}$ is a function of $C$ from (2.2). Assume that there is a discontinuous curve $x=x(t)$ which is first-order continuously differentiable and $p>p_{r}$ everywhere, namely, it is a forward shock wave. From $(2.1)$ it is easy to obtain

$$
d x / d t=\sqrt{\left[\left(\gamma_{c, r}+1\right) p+\left(\gamma_{c, r}-1\right) p_{r}\right] / 2 v_{r}} .
$$

By simple calculation we have

LEMMA 1. If $\gamma_{1}, \gamma_{2} \leqslant \frac{5}{3}, u_{r}, p_{r}, v_{r}, \gamma_{c, r}$ in (2.13) are continuously differentiable functions of $t$, and functions $u=u(t), p=p(t)$ satisfy (2.13). Then

$$
\frac{d u}{d t}+l_{12}(p, c, s) \frac{d p}{d t}=\beta\left(\frac{d u}{d t}+l_{42}(p, c, s) \frac{d p}{d t}\right)+G(p, t)
$$

where $0 \leqslant \beta<1$, and $G$ is a first-order continuously differentiable function of $p$ and $t$.

3. Riemann problem. We discuss the following problem in this section.

$$
\begin{aligned}
& \partial v / \partial t-\partial u / \partial x=0, \quad \partial c / \partial t=0, \quad \partial u / \partial t+\partial p / \partial x=0, \\
& \frac{\partial}{\partial t}\left(\frac{p v}{\gamma_{c}-1}+\frac{u^{2}}{2}+c q_{1}+(1-c) q_{2}\right)+\frac{\partial}{\partial x}(p u)=0 . \\
& (v(x, 0), c(x, 0), u(x, 0), p(x, 0))= \begin{cases}\left(v_{l}, c_{l}, u_{l}, p_{l}\right), & x<0, \\
\left(v_{r}, c_{r}, u_{r}, p_{r}\right), & x>0,\end{cases}
\end{aligned}
$$

where (3.1) is the homogeneous system corresponding to $(2.1), v_{l}, \ldots, p_{r}$ are all constants, $p_{l}>0, p_{r}>0,0 \leqslant c_{l} \leqslant 1,0 \leqslant c_{r} \leqslant 1$.

THEOREM 1. If

$$
u_{r}-\frac{2 \sqrt{\gamma_{c, r}}}{\gamma_{c, r}-1} v_{r}^{1 / 2} p_{r}^{1 / 2}<u_{l}+\frac{2 \sqrt{\gamma_{c, l}}}{\gamma_{c, l}-1} v_{l}^{1 / 2} p_{l}^{1 / 2}
$$


then the solution of problem (3.1)-(3.2) exists at the region $t>0,-\infty<x<+\infty$, and $p>0,0 \leqslant c \leqslant 1$. Moreover, the structure of the solution is:

(a) at the t-axis, functions $u$ and $p$ are continuous, but $c$ and $v$ are discontinuous in general-a double characteristic contact discontinuity;

(b) forward wave;

(c) backward wave;

(d) constants at the rest of the region;

where the forward and backward waves may be shock or rarefaction waves. The rarefaction wave is continuous at each point except the origin; the forward shock wave satisfies conditions (2.13)-(2.16) and the entropy condition $u>u_{r}$, the backward shock wave satisfies some similar conditions.

Proof. We draw curves on the $(u, p)$ plane

$$
\begin{aligned}
& L_{1}: u-u_{l}=\left\{\begin{array}{l}
-\left(p-p_{l}\right)\left\{\frac{2 v_{l}}{\left(\gamma_{c, l}+1\right) p+\left(\gamma_{c, l}-1\right) p_{l}}\right\}^{1 / 2}, \quad p>p_{l}, \\
-\frac{2 \sqrt{\gamma_{c, l}}}{\gamma_{c, l}-1} v_{l}^{1 / 2} p_{l}^{1 / 2 \gamma_{c, l}}\left(p^{\left(\gamma_{c, l}-1\right) / 2 \gamma_{c, l}}-p \gamma^{\left.\left(\gamma_{c, l}-1\right) / 2 \gamma_{c, l}\right),} \quad p<p_{l},\right.
\end{array}\right.
\end{aligned}
$$

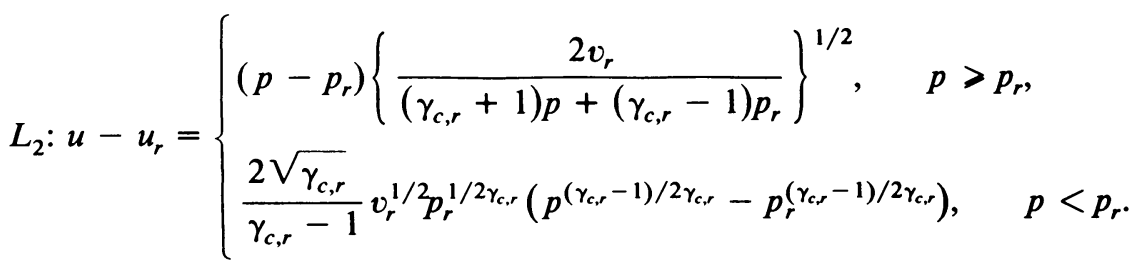

It is not difficult to obtain by inspection that

$$
L_{1}: d u / d p<0, \quad d^{2} u / d p^{2}>0, \quad L_{2}: d u / d p>0, \quad d^{2} u / d p^{2}<0 .
$$

Hence, there must be a unique intersection point $(\bar{u}, \bar{p})$.

If $\bar{p} \geqslant p_{r}$, the forward wave is a shock wave; We can determine $\bar{c}$ and $\bar{v}$ by (2.14)-(2.15). If $\bar{p}<p_{r}$, the forward wave is a rarefaction wave; we can determine $\bar{c}$ and $\bar{v}$ by

$$
\bar{p} \bar{v}^{\gamma_{c, r}}=p_{r} v_{r}^{\gamma_{c, r}}, \quad \bar{c}=c_{r} .
$$

Similarly, we can determine $\hat{c}$ and $\hat{v}$ in case $\bar{p} \geqslant p_{l}$ or $\bar{p}<p_{l}$. We obtain four constant regions which are connected by waves as follows:

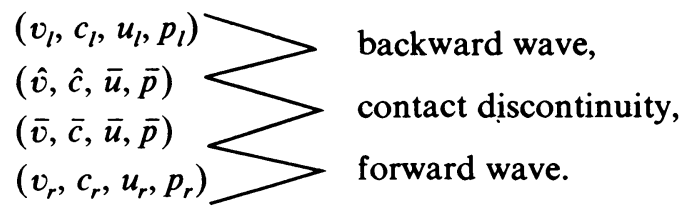

We can get $\bar{p}>0$ from (3.3); as for $\bar{c}, \hat{c} \in[0,1]$, it is obvious. Q.E.D. 
4. Rarefaction wave. From (2.12), (3.1) can be written as

$$
l_{i}(r)\left(d r / d t_{i}\right)=0, \quad(i=1,2,3,4) .
$$

Taking the forward wave as an example, we notice that the boundary value conditions satisfied by a rarefaction wave are the following:

$$
\begin{array}{ll}
\left.r\right|_{x=a_{1} t}=r_{1}, & \left.\lambda_{4}(r)\right|_{x=a_{1} t}=a_{1}, \\
\lim _{x=\theta t, t \rightarrow 0} \lambda_{4}(r)=\theta, & \theta \in\left[a_{0}, a_{1}\right],
\end{array}
$$

where $r_{1}, a_{0}, a_{1}$ are constants. There are similar boundary value conditions for backward rarefaction waves.

Now we define the (forward) rarefaction wave of (2.12). Let the vector function $r(x, t)$ satisfy $(2.12)$ and the following:

$$
\begin{array}{lll}
L: x=x(t), & d x / d t=\lambda_{4}(r), & \left.(d x / d t)\right|_{t=0}=a_{1}, \\
\left.r\right|_{L}=\phi(t), & \left.l_{4}(r)(d r / d t)\right|_{L}=\left.b_{4}(r)\right|_{L}, & \\
\lim _{x=\theta t, t \rightarrow 0} \lambda_{4}(r)=\theta, & \theta \in\left[a_{0}, a_{1}\right]
\end{array}
$$

where $L$ is a curve in the $(x, t)$ plane; then $r(x, t)$ is called a forward rarefaction wave. We prove the local existence of (2.12), (4.3) provided the solution of (4.1), (4.2) exists. In order to distinguish them, the solution of (4.1), (4.2) is denoted by $r^{(0)}$, which is a self-similar solution from $\S 3$, namely, $r^{(0)}(\alpha x, \alpha t)=r^{(0)}(x, t)$, where $\alpha$ is a positive constant.

$r^{(0)}$ is a constant vector on the line $x=\theta t$, hence $r^{(0)}$ can be looked upon as a function of $\theta$; replacing $\theta$ we introduce a new independent variable,

$$
\beta=\beta(\theta)=r_{4}^{(0)}(\theta)
$$

It is obvious that

Lemma 2. There is a one-to-one correspondence between $\beta$ and $\theta$.

We introduce the function $x=x(\theta, t)$ which satisfies the following initial value problem of ordinary differential equations:

$$
\begin{aligned}
& \partial x / \partial t=\lambda_{4}(r(x, t)),\left.x\right|_{t=0}=0 \\
& \left.(\partial x / \partial t)\right|_{t=0}=\theta, \quad \theta \in\left[a_{0}, a_{1}\right] .
\end{aligned}
$$

From (4.3) we know that the origin is a singular point of this equation, so we use two initial values, and it is not difficult to prove that the solution of (4.5) exists, and is unique, provided $r(x, t)$ exists and satisfies the Lipschitz condition on each closed region excluding the origin.

Let

$$
\begin{aligned}
& \beta_{0}=\beta\left(\theta_{0}\right), \quad \beta_{1}=\beta\left(\theta_{1}\right), \quad X(\beta, t)=x(\theta(\beta), t), \\
& R(\beta, t)=r(X(\beta, t), t) .
\end{aligned}
$$


From (2.12) and (4.3), the equations and boundary values can be written as

$$
\begin{gathered}
l_{i}(r)\left(d r / d t_{i}\right)=b_{i}(r), \quad(i=1,2,3), \\
l_{4}(r)(\partial R / \partial t)=b_{4}(r), \\
R\left(\beta_{1}, t\right)=\phi(t), \quad l_{4}(\phi(t)) \phi^{\prime}(t)=b_{4}(\phi(t)), \\
R_{4}(\beta, 0)=\beta, \quad \beta \in\left[\beta_{0}, \beta_{1}\right] .
\end{gathered}
$$

We are going to prove the local existence of the solutions of problem (4.5), (4.6); from $\S 2, l_{i}(r), \lambda_{i}(r)(i=1,2,3,4)$ are sufficiently smooth and (2.12) is hyperbolic, namely, the determinant

$$
D=\operatorname{det}\left(\begin{array}{l}
l_{1}(r) \\
l_{2}(r) \\
l_{3}(r) \\
l_{4}(r)
\end{array}\right)
$$

is nonsingular. Now we suppose functions $b_{i}(r)(i=1,2,3,4), \phi(t)$ satisfy the Lipschitz condition of their domains of definition, respectively.

Take the monotone sequence $\left\{\tau_{n}\right\}(n=1,2, \ldots)$ such that $\tau_{n} \rightarrow 0(n \rightarrow \infty)$, take the sequences $\left\{b_{i}^{(n)}(r, t)\right\}(i=1,2,3,4, n=1,2, \ldots)$ and $\left\{\phi^{(n)}(t)\right\}(n=$ $1,2, \ldots)$ such that they converge uniformly to $b_{i}(r)$ and $\phi(t)$ as $n \rightarrow \infty$; they are first-order continuously differentiable and $\partial b_{i}^{(n)} / \partial r_{j}, d \phi^{(n)} / d t$ are bounded uniformly; $b_{i}^{(n)}(r, t) \equiv 0, \phi^{(n)}(t) \equiv \phi(0)$ for $t \leqslant \tau_{n}$; besides

$$
l_{4}\left(\phi^{(n)}(t)\right)\left(d \phi^{(n)}(t) / d t\right)=b_{4}^{(n)}\left(\phi^{(n)}(t), t\right) .
$$

It is easy to show that the following lemma holds.

LEMMA 3. The sequences $\left\{b_{i}^{(n)}(r, t)\right\}$ an $\left\{\phi^{(n)}(t)\right\}$ exist.

Substituting $b_{i}^{(n)}(r, t)$ and $\phi^{(n)}(t)$ for $b_{i}(r)$ and $\phi(t)$ in (4.6), we get a new problem compared with problem (4.5), (4.6) which is called $(4.5)_{n},(4.6)_{n}$. The solutions of $(4.5)_{n}$ and (4.6) $)_{n}$ are denoted by $x^{(n)}(\theta, t), r^{(n)}(x, t), R^{(n)}(\beta, t)$ and so on.

LEMMA 4. There exists $\tau_{n}^{\prime}>\tau_{n}$, such that problem $(4.5)_{n},(4.6)_{n}$ has smooth solutions as $t \in\left[0, \tau_{n}^{\prime}\right)$.

Proof. We take $r^{(n)}(x, t) \equiv r^{(0)}(x, t), x^{(n)}(\theta, t) \equiv \theta t$ for $t<\tau_{n}$, where $r_{1}=\phi(0)$; it is obvious that they satisfy $(4.5)_{n},(4.6)_{n}$. For $t>\tau_{n}$, we take $r^{(0)}\left(x, \tau_{n}\right)$ as the initial value; from [9] the smooth solutions exist on a suitable region. Q.E.D.

In the following we let $K$ be a constant depending only on the upper and lower bounds of functions $l_{i}(r), \lambda_{i}(r), b_{i}^{(n)}(r, t), \phi^{(n)}(t)$, and the upper and lower bounds of derivatives $\partial l_{i}(r) / \partial r_{j}, \partial \lambda_{i}(r) / \partial r_{j}, \partial b_{i}^{(n)} / \partial r_{j}, d \phi^{(n)} / d t$, and constants $a_{0}, a_{1} . K$ could be taken as different values at various times, but the relation of dependence is known. However, we usually consider $K$ as the maximal value under many conditions. 
LEMMA 5. There exist constants $\tau_{0}>0, M_{0}, M_{1}, M_{2}, m_{2}>0$, depending only on $K$, such that for $t \in\left(0, \tau_{0}\right]$, the smooth solutions of problem $(4.5)_{n},(4.6)_{n}$ exist and satisfy the following estimation:

$$
\begin{gathered}
\left|r^{(n)}\right| \leqslant M_{0}, \\
\left|\partial r_{i}^{(n)} / \partial x\right|,\left|\partial r_{i}^{(n)} / \partial t\right| \leqslant M_{1}, \quad(i=1,2,3), \\
\left|\partial R^{(n)} / \partial t\right| \leqslant M_{1}, \\
m_{2} \leqslant\left|\frac{\partial R_{4}^{(n)}}{\partial \beta}\right| \leqslant M_{2}, \quad t\left|\frac{\partial r_{4}^{(n)}}{\partial x}\right| \leqslant M_{2}, \quad t\left|\frac{\partial r_{4}^{(n)}}{\partial t}\right| \leqslant M_{2} .
\end{gathered}
$$

Proof. The constants $\tau_{0}, M_{0}, M_{1}, M_{2}, m_{2}$ will be given gradually. The inequality

$$
D^{(n)}=\operatorname{det}\left[\begin{array}{l}
l_{1}\left(r^{(n)}\right) \\
l_{2}\left(r^{(n)}\right) \\
l_{3}\left(r^{(n)}\right) \\
l_{4}\left(r^{(n)}\right)
\end{array}\right] \geqslant \frac{1}{K}>0
$$

holds as (4.8) holds. We know $r^{(n)} \equiv r^{(0)}$ for each solution $r^{(n)}$ when $t \leqslant \tau_{n}$, hence $r_{i}^{(n)} \equiv$ const. $(i=1,2,3)$ and $R_{4}^{(n)}(\beta, t) \equiv \beta$, therefore inequalities (4.8)-(4.11) always hold for $t \leqslant \tau_{n}$ provided we take suitable $M_{0}, M_{2}$. By the smoothness of the solutions, a constant $\tau_{n}^{\prime}>\tau_{n}$ exists such that these inequalities hold as $t \in\left(0, \tau_{n}^{\prime}\right]$ for each solution. For now we will only consider the case $t \in\left(0, \tau_{n}^{\prime}\right]$.

For $i=1,2,3$, let $\xi(\eta, x, t)$ satisfy the following initial value problem of ordinary differential equations:

$$
\partial \xi / \partial \eta=\lambda_{i}\left(r^{(n)}(\xi, \eta)\right),\left.\quad \xi\right|_{\eta=t}=x .
$$

The function $\xi=\xi(\eta, x, t)$ denotes a characteristic curve passing through the point $(x, t)$; suppose it intersects the curve $X\left(\beta_{1}, t\right)$ at $t=\tau$, namely

$$
\xi(\tau, x, t)=X\left(\beta_{1}, \tau\right) \text {. }
$$

We integrate the $i$ th equation of (4.6) $)_{n}$ along the characteristic curve and obtain

$$
\begin{aligned}
& \left.l_{i}\left(r^{(n)}\right) r^{(n)}\right|_{(x, t)}=\left.l_{i}\left(r^{(n)}\right) r^{(n)}\right|_{(\xi(\tau, x, t), \tau)} \\
& +\int_{\tau}^{t}\left[b_{i}^{(n)}\left(r^{(n)}(\xi(\eta, x, t), \eta), \eta\right)+\frac{\partial}{\partial \eta} l_{i}\left(r^{(n)}(\xi(\eta, x, t), \eta)\right) r^{(n)}(\xi(\eta, x, t), \eta)\right] d \eta \\
& (i=1,2,3) .
\end{aligned}
$$

We have

$$
\begin{gathered}
\left|l_{i}\left(r^{(n)}\right) r^{(n)}\right|_{(\xi(\tau, x, t), \tau)} \leqslant K, \quad\left|b_{i}^{(n)}\right| \leqslant K, \\
\left|\frac{\partial l_{i}}{\partial \eta}\right|=\left|\frac{\partial l_{i}}{\partial r_{j}}\left(\frac{\partial r_{j}^{(n)}}{\partial x} \frac{\partial \xi}{\partial \eta}+\frac{\partial r_{j}^{(n)}}{\partial t}\right)\right| \leqslant K\left(M_{1}+\frac{M_{2}}{t}\right) .
\end{gathered}
$$

Let

$$
U(t)=\max _{i=1,2,3}\left|r_{i}^{(n)}(x, t)\right|
$$


Then from (4.15),

$$
\left|l_{i}\left(r^{(n)}\right) r^{(n)}\right| \leqslant K+\int_{\tau}^{t}\left[K+K\left(M_{1}+\frac{M_{2}}{t}\right) U(t)\right] d t, \quad(i=1,2,3) .
$$

Here we indicate that we have used a property of $l_{i}$, namely, the fourth components vanish, hence $r_{4}^{(n)}$ does not appear within the integral. Regarding (4.12), we get

$$
\left|r_{i}^{(n)}\right| \leqslant K+\int_{\tau}^{t} K\left[1+\left(M_{1}+\frac{M_{2}}{t}\right) U(t)\right] d t, \quad(i=1,2,3) .
$$

Taking the maximum with respect to $i$ and $x$, we get

$$
U(t) \leqslant K+\int_{\tau}^{t} K\left[1+\left(M_{1}+\frac{M_{2}}{t}\right) U(t)\right] d t,
$$

where $\tau$ denotes the infimum of $\tau$ corresponding to each $x$. Therefore,

$$
\begin{aligned}
\left|r_{i}^{(n)}(x, t)\right| \leqslant & K\left(K M_{2}-1\right)^{-1} e^{K M_{1} t}(t / \tau)^{K M_{2}-1} t \\
& +K e^{K M_{1}(t-\tau)}(t / \tau)^{K M_{2}} \quad(i=1,2,3) .
\end{aligned}
$$

From (4.13),

$$
\frac{\partial}{\partial \eta}\left(\frac{\partial \xi}{\partial x}\right)=\frac{\partial \lambda_{i}}{\partial r_{j}} \frac{\partial r_{j}}{\partial x} \frac{\partial \xi}{\partial x},\left.\quad \frac{\partial \xi}{\partial x}\right|_{\eta=t}=1
$$

Hence

$$
\frac{\partial \xi}{\partial x}=\exp \left\{\int_{t}^{\eta} \frac{\partial \lambda_{i}}{\partial r_{j}} \frac{\partial r_{j}^{(n)}}{\partial x} d \eta\right\} \leqslant \exp \left\{\int_{\eta}^{t} K\left(M_{1}+\frac{M_{2}}{\eta}\right) d \eta\right\}
$$

namely

$$
0<\partial \xi / \partial x \leqslant e^{K M_{1}(t-\eta)}(t / \eta)^{K M_{2}}
$$

We know that $\tau$ is the function of $x, t$ in (4.14). Now we look on $\tau$ as a constant in (4.15), namely $(\xi(\tau, x, t), \tau)$ need not be a boundary point. Differentiate (4.15) with respect to $x$, and it is easy to prove that

$$
\begin{aligned}
l_{i}\left(r^{(n)}\right) \frac{\partial r^{(n)}}{\partial x}= & \left.l_{i}\left(r^{(n)}\right) \frac{\partial r^{(n)}}{\partial x}\right|_{(\xi(\tau, x, t), \tau)} \\
& +\int_{\tau}^{t}\left[\frac{\partial b_{i}^{(n)}\left(r^{(n)}, \eta\right)}{\partial x}+\frac{\partial l_{i}\left(r^{(n)}\right)}{\partial \eta} \frac{\partial r^{(n)}}{\partial x}-\frac{\partial l_{i}\left(r^{(n)}\right)}{\partial x} \frac{\partial r^{(n)}}{\partial \eta}\right] d \eta
\end{aligned}
$$

where

$$
\begin{gathered}
\left.\frac{\partial r^{(n)}}{\partial x}\right|_{(\xi(\tau, x, t), \tau)}=\left.\frac{\partial r^{(n)}(x, t)}{\partial x}\right|_{x=\xi(\tau, x, t)} \cdot \frac{\partial \xi(\tau, x, t)}{\partial x}, \\
\partial b_{i}^{(n)}\left(r^{(n)}, \eta\right) / \partial x=\left.\frac{\partial b_{i}^{(n)}(r, \eta)}{\partial r_{j}} \frac{\partial r_{j}^{(n)}(x, t)}{\partial x} \frac{\partial \xi(\tau, x, t)}{\partial x}\right|_{\substack{r=r^{(n)}(\xi(\eta, x, t), \eta) \\
x=\xi(\eta, x, t)}}
\end{gathered}
$$


The other terms in the integration must be understood similarly; we rewrite (4.18) in detail as

$$
\begin{aligned}
\left.l_{i}\left(r^{(n)}\right)\left(\partial r^{(n)} / \partial x\right)\right|_{t}= & \left.l_{i}\left(r^{(n)}\right)\left(\partial r^{(n)} / \partial x\right)(\partial \xi / \partial x)\right|_{\tau} \\
& +\int_{\tau}^{t}\left[\frac{\partial b_{i}^{(n)}}{\partial r_{j}} \frac{\partial r_{j}^{(n)}}{\partial x} \frac{\partial \xi}{\partial x}\right. \\
& +\frac{\partial l_{i}}{\partial r_{j}}\left(\frac{\partial r_{j}^{(n)}}{\partial x} \frac{\partial \xi}{\partial \eta}+\frac{\partial r_{j}^{(n)}}{\partial t}\right) \frac{\partial r^{(n)}}{\partial x} \frac{\partial \xi}{\partial x} \\
& \left.-\frac{\partial l_{i}}{\partial r_{j}} \frac{\partial r_{j}^{(n)}}{\partial x} \frac{\partial \xi}{\partial x}\left(\frac{\partial r^{(n)}}{\partial x} \frac{\partial \xi}{\partial \eta}+\frac{\partial r^{(n)}}{\partial t}\right)\right] d \eta
\end{aligned}
$$

where the $j$ 's repeating in one term means taking the sum $(j=1,2,3,4)$. We note that the fourth components of $l_{i}$ vanish, so $\partial r^{(n)} / \partial x$ actually has only its first three components. We know from (4.6) $n$ that as (4.14) holds, namely, when the point is located at the boundary, then

$$
\frac{\partial r_{j}^{(n)}}{\partial t}+\lambda_{4}\left(r^{(n)}\right) \frac{\partial r_{j}^{(n)}}{\partial x}=\frac{d}{d t} \phi^{(n)}(t), \quad(j=1,2,3) .
$$

Combining this with the differential equations

$$
l_{j}\left(r^{(n)}\right)\left(\frac{\partial r^{(n)}}{\partial t}+\lambda_{j}\left(r^{(n)}\right) \frac{\partial r^{(n)}}{\partial x}\right)=b_{j}^{(n)}\left(r^{(n)}, t\right), \quad(j=1,2,3),
$$

we can solve for the values of $\partial r_{j}^{(n)} / \partial t$ and $\partial r_{j}^{(n)} / \partial x$ at the boundary and obtain

$$
\left|\left(\partial r_{j}^{(n)} / \partial x\right)_{\beta=\beta_{1}}\right| \leqslant K, \quad(j=1,2,3) .
$$

We also use the fact that the fourth components of $l_{i}$ vanish for the terms under integration and note (4.17); then we obtain

$$
\begin{aligned}
& \left|l_{i}\left(r^{(n)}\right)\left(\partial r^{(n)} / \partial x\right)\right| \leqslant K e^{K M_{1}(t-\tau)}(t / \tau)^{K M_{2}} \\
& +K \int_{\tau}^{t} e^{K M_{1}(t-\eta)}\left(\frac{t}{\eta}\right)^{K M_{2}}\left[M_{1}+\frac{M_{2}}{\eta}+\left(M_{1}+\frac{M_{2}}{\eta}\right)\right. \\
& \left.\cdot \max _{j=1,2,3}\left\{\left|\frac{\partial r_{j}^{(n)}}{\partial x}\right|,\left|\frac{\partial r_{j}^{(n)}}{\partial t}\right|\right\}\right] d \eta .
\end{aligned}
$$

From $(4.6)_{n}$ we have

$$
l_{i}\left(r^{(n)}\right)\left(\frac{\partial r^{(n)}}{\partial t}+\lambda_{i}\left(r^{(n)}\right) \frac{\partial r^{(n)}}{\partial x}\right)=b_{i}^{(n)}\left(r^{(n)}, t\right)
$$

Left multiplying it by the inverse matrix, we obtain

$$
\begin{aligned}
\left|\frac{\partial r_{i}^{(n)}}{\partial t}\right| \leqslant K\left(1+\max _{j=1,2,3}\left|\frac{\partial r_{j}^{(n)}}{\partial x}\right|\right), \quad(i=1,2,3), & \left.\left|\frac{\partial r_{i}^{(n)}}{\partial x}\right|,\left|\frac{\partial r_{i}^{(n)}}{\partial t}\right| \leqslant \mid K e^{K M_{1}(t-\tau)}\left(\frac{t}{\tau}\right)^{K M_{2}}+1\right] \\
& \cdot\left\{e^{M_{1}(t-\tau)}\left(\frac{t}{\tau}\right)^{M_{2}}\right\}^{K \exp \left\{K M_{1}(t-\tau)\right\}(t / \tau)^{K M_{2}}}, \quad(i=1,2,3) .
\end{aligned}
$$


Now we estimate $r_{4}^{(n)}$; from (4.6) ,

$$
\begin{aligned}
l_{4}\left(R^{(n)}(\beta, t)\right) R^{(n)}(\beta, t) & =l_{4}\left(R^{(n)}(\beta, 0)\right) R^{(n)}(\beta, 0) \\
+ & \int_{0}^{t}\left[b_{4}^{(n)}\left(R^{(n)}(\beta, \eta), \eta\right)+\frac{\partial}{\partial \eta} l_{4}\left(R^{(n)}(\beta, \eta)\right) R^{(n)}(\beta, \eta)\right] d \eta
\end{aligned}
$$

from (4.10),

$$
\left|l_{4}\left(R^{(n)}\right) R^{(n)}\right| \leqslant K+K\left(1+M_{0} M_{1}\right) t .
$$

Combining this with (4.16), we obtain

$$
\begin{aligned}
\left|r^{(n)}\right| \leqslant & K+K\left[\frac{1}{K M_{2}-1} e^{K M_{1} t}\left(\frac{t}{\tau}\right)^{K M_{2}-1}+1+M_{0} M_{1}\right] t \\
& +K e^{K M_{1}(t-\tau)}(t / \tau)^{K M_{2}} .
\end{aligned}
$$

From (4.23), $\left|\partial R_{i}^{(n)}(\beta, t) / \partial t\right|(i=1,2,3)$ can be estimated and the bound is the same as (4.23). We notice $(4.6)_{n}$,

$$
l_{4}\left(R^{(n)}\right)\left(\partial R^{(n)} / \partial t\right)=b_{4}^{(n)}\left(R^{(n)}, t\right),
$$

hence for $i=1,2,3,4$, we can get

$$
\begin{aligned}
\left|\frac{\partial R_{i}^{(n)}(\beta, t)}{\partial t}\right| \leqslant & K+\left[K e^{K M_{1}(t-\tau)}\left(\frac{t}{\tau}\right)^{K M_{2}}+1\right] \\
& \cdot\left\{e^{M_{1}(t-\tau)}\left(\frac{t}{\tau}\right)^{M_{2}}\right\}^{K \exp \left\{K M_{1}(t-\tau)\right\}(t / \tau)^{K M_{2}}} .
\end{aligned}
$$

Differentiating (4.5) $)_{n}$ with respect to $\beta$, we obtain

$$
\frac{\partial}{\partial t}\left(\frac{\partial X^{(n)}}{\partial \beta}\right)=\sum_{j=1}^{3} \frac{\partial \lambda_{4}}{\partial r_{j}} \frac{\partial r_{j}^{(n)}}{\partial x} \frac{\partial X^{(n)}}{\partial \beta}+\frac{\partial \lambda_{4}}{\partial r_{4}} \frac{\partial R_{4}^{(n)}}{\partial \beta},\left.\quad \frac{\partial X^{(n)}}{\partial \beta}\right|_{t=0}=0 ;
$$

hence,

$$
\begin{aligned}
\frac{\partial X^{(n)}}{\partial \beta}= & \int_{0}^{t} \frac{\partial \lambda_{4}}{\partial r_{4}} \frac{\partial R_{4}^{(n)}}{\partial \beta} \exp \left\{-\int_{0}^{\eta} \sum_{j=1}^{3} \frac{\partial \lambda_{4}}{\partial r_{j}} \frac{\partial r_{j}^{(n)}}{\partial x} d \eta^{\prime}\right\} d \eta \\
& \cdot \exp \left\{\int_{0}^{t} \sum_{j=1}^{3} \frac{\partial \lambda_{4}}{\partial r_{j}} \frac{\partial r_{j}^{(n)}}{\partial x} d \eta\right\} .
\end{aligned}
$$

From the expressions of systems in $\$ 2$ we find

$$
\left|\partial \lambda_{4} / \partial r_{4}\right| \geqslant 1 / K>0 \text {. }
$$

From (4.9), (4.11) and (4.27),

$$
K^{-1} m_{2} t e^{-K M_{1} t} \leqslant\left|\partial X^{(n)} / \partial \beta\right| \leqslant K M_{2} t e^{K M_{1} t} .
$$

Differentiating (4.24) with respect to $\beta$, noting $(2.11)$ and $\partial R_{j}^{(n)}(\beta, 0) / \partial \beta=0$ $(j=1,2,3), R_{4}^{(n)}(\beta, 0)=\beta$, we obtain

$$
\begin{aligned}
& l_{4}\left(R^{(n)}(\beta, t)\right) {\left[\partial R^{(n)}(\beta, t) / \partial \beta\right] } \\
&=1+\int_{0}^{t}\left[\frac{\partial b_{4}^{(n)}}{\partial R_{j}} \frac{\partial R_{j}^{(n)}}{\partial \beta}+\frac{\partial l_{4}}{\partial R_{j}} \frac{\partial R_{j}^{(n)}}{\partial \eta} \frac{\partial R^{(n)}}{\partial \beta}-\frac{\partial l_{4}}{\partial R_{j}} \frac{\partial R_{j}^{(n)}}{\partial \beta} \frac{\partial R^{(n)}}{\partial \eta}\right] d \eta .
\end{aligned}
$$


For $j=1,2,3$,

$$
\frac{\partial R_{j}^{(n)}}{\partial \beta}=\frac{\partial r_{j}^{(n)}}{\partial x} \frac{\partial X^{(n)}}{\partial \beta}
$$

from (4.9) and (4.28),

$$
\left|\partial R_{j}^{(n)} / \partial \beta\right|<K M_{1} M_{2} t e^{K M_{1} t}, \quad(j=1,2,3) .
$$

From (4.10) and (4.11), the function under integration satisfies

Hence,

$$
\begin{aligned}
\mid \frac{\partial b_{4}^{(n)}}{\partial R_{j}} \frac{\partial R_{j}^{(n)}}{\partial \beta} & +\frac{\partial l_{4}}{\partial R_{j}} \frac{\partial R_{j}^{(n)}}{\partial \eta} \frac{\partial R^{(n)}}{\partial \beta}-\frac{\partial l_{4}}{\partial R_{j}} \frac{\partial R_{j}^{(n)}}{\partial \beta} \frac{\partial R^{(n)}}{\partial \eta} \mid \\
& <K\left(1+M_{1}\right)\left(M_{2}+K M_{1} M_{2} \eta e^{K M_{1} \eta}\right) .
\end{aligned}
$$

$$
\left|l_{4}\left(R^{(n)}(\beta, t)\right)\left[\partial R^{(n)}(\beta, t) / \partial \beta\right]-1\right| \leqslant K\left(1+M_{1}\right)\left(M_{2}+K M_{1} M_{2} t e^{K M_{1} t}\right) t
$$

We use (4.29) again and obtain

$$
\left|\partial R_{4}^{(n)}(\beta, t) / \partial \beta-1\right| \leqslant K\left(1+M_{1}\right)\left(M_{2}+K M_{1} M_{2} t e^{K M_{1} t}\right) t .
$$

Because

$$
\frac{\partial r_{4}^{(n)}(x, t)}{\partial x}=\frac{\partial R_{4}^{(n)}(\beta, t) / \partial \beta}{\partial X^{(n)} / \partial \beta}
$$

we obtain from (4.28) and (4.30),

$$
\left|\frac{\partial r_{4}^{(n)}}{\partial x}\right| \leqslant \frac{K}{m_{2} t}\left\{1+K\left(1+M_{1}\right)\left(M_{2}+K M_{1} M_{2} t e^{K M_{1} t}\right) t\right\} .
$$

From $(4.6)_{n}$,

$$
\left|\frac{\partial r_{4}^{(n)}}{\partial t}\right|<K\left\{\sum_{j=1}^{3}\left(\left|\frac{\partial r_{j}^{(n)}}{\partial x}\right|+\left|\frac{\partial r_{j}^{(n)}}{\partial t}\right|\right)+\left|\frac{\partial r_{4}^{(n)}}{\partial x}\right|+1\right\} .
$$

From (4.23) and (4.31),

$$
\begin{aligned}
t\left|\partial r_{4}^{(n)} / \partial t\right| \leqslant & K t\left[K e^{K M_{1}(t-\tau)}(t / \tau)^{K M_{2}}+1\right] \\
& \cdot\left\{e^{M_{1}(t-\tau)}(t / \tau)^{M_{2}}\right\}^{K \exp \left\{K(t-\tau)(t / \tau)^{K M_{2}}\right\}} \\
& +\left(K / m_{2}\right) e^{K M_{1} t}\left\{1+K\left(1+M_{1}\right)\left(M_{2}+K M_{1} M_{2} t e^{K M_{1} t}\right) t\right\}+K t
\end{aligned}
$$

We know from the expressions in $\S 2$, that $\lambda_{4}(r)>1 / K>0$ on a bounded region; hence

$$
0<t / \tau \leqslant \max \lambda_{4}(r) / \min \lambda_{4}(r) \leqslant K .
$$

We compare (4.25), (4.23), (4.26), (4.30)-(4.32) and (4.8)-(4.11) and determine the constants $M_{0}, M_{1}, M_{2}, m_{2}, \tau_{0}$ gradually as follows.

(a) Take $m_{2}, 0<m_{2}<1$; for instance, take $m_{2}=\frac{1}{2}$.

(b) Take $M_{2}=\max \left\{3 / 2,3 K / m_{2}+K\right\}$.

(c) Take $M_{1}=K+\left[2 K K^{K M_{2}}+1\right]\left\{2 K^{M_{2}}\right\}^{2 K K^{K M_{2}}}$.

(d) Take $M_{0}=K+1+2 K K^{K M_{2}}$. 
(e) Take $\tau_{0}$ such that

$$
\begin{aligned}
e^{K M_{1} \tau_{0}} & <2, \\
K\left[\frac{2}{K M_{2}-1} K^{K M_{2}-1}+1+M_{0} M_{1}\right] \tau_{0} & \leqslant 1, \\
K\left(1+M_{1}\right)\left(M_{2}+2 K M_{1} M_{2} \tau_{0}\right) \tau_{0} & \leqslant 1 / 2, \\
\tau_{0}\left[2 K K^{K M_{2}}+1\right]\left\{2 K^{M_{2}}\right\}^{2 K K^{K M_{2}}}+\tau_{0} & <1 .
\end{aligned}
$$

At the beginning of this lemma we have assumed that inequalities (4.8)-(4.11) hold on a domain $\left\{(x, t) \mid t \in\left[0, \tau_{n}^{\prime}\right], X^{(n)}\left(\beta_{0}, t\right) \leqslant x \leqslant X^{(n)}\left(\beta_{1}, t\right)\right\}$. Suppose $\tau_{n}^{\prime}$ is the supremum of such values; then at least one equality holds among these inequalities on this closed domain, because if all of the equalities did not hold, we could solve it on a larger domain by means of the local solvability, and $\tau_{n}^{\prime}$ would not be the supremum.

We prove $\tau_{n}^{\prime} \geqslant \tau_{0}$; if not, $\tau_{n}^{\prime}<\tau_{0}$; from the way of choosing $M_{0}, M_{1}, M_{2}, m_{2}, \tau_{0}$, the upper and lower bounds in (4.8)-(4.11) could not be achieved on the domain $\left\{(x, t) \mid t \in\left[0, \tau_{n}^{\prime}\right], X^{(n)}\left(\beta_{0}, t\right) \leqslant x \leqslant X^{(n)}\left(\beta_{1}, t\right)\right\}$, a contradiction. Q.E.D.

THEOREM 2. If problem (4.5), (4.6) satisfies the above conditions and the function $f(v, p, c)$ in (2.1) satisfies

$$
\begin{aligned}
& f(v, p, c) \geqslant 0 \quad \text { for } c \leqslant 0, \\
& f(v, p, c) \leqslant 0 \quad \text { for } c \geqslant 1,
\end{aligned}
$$

then there exists a constant $\tau_{0}>0$, such that functions $x(\theta, t), r(x, t)$ exist: $t \in$ $\left[0, \tau_{0}\right], \theta_{0} \leqslant \theta \leqslant \theta_{1}, X\left(\beta_{0}, t\right) \leqslant x \leqslant X\left(\beta_{1}, t\right)$, the functions satisfy the Lipschitz condition and satisfy (4.5)-(4.6) almost everywhere for $t \in\left[\tau, \tau_{0}\right], \tau_{0}>0$. Moreover,

$$
\begin{aligned}
&|r| \leqslant M_{0}, \quad\left|\frac{\partial r_{i}}{\partial x}\right|,\left|\frac{\partial r_{i}}{\partial t}\right| \leqslant M_{1}(i=1,2,3), \quad\left|\frac{\partial R(\beta, t)}{\partial t}\right| \leqslant M_{1}, \\
& m_{2} \leqslant\left|\frac{\partial R_{4}}{\partial \beta}\right| \leqslant M_{2}, \quad t\left|\frac{\partial r_{4}}{\partial x}\right| \leqslant M_{2}, \quad t\left|\frac{\partial r_{4}}{\partial t}\right|<M_{2}, \\
& p \geqslant \delta>0, \\
& 0 \leqslant c \leqslant 1,
\end{aligned}
$$

where constants $\tau_{0}, M_{0}, M_{1}, M_{2}, m_{2}, \delta$ depend only on the upper and lower bounds of $l_{i}(r), \lambda_{i}(r), b_{i}(r), \phi(t), \partial l_{i}(r) / \partial r_{j}, \partial \lambda_{i}(r) / \partial r_{j}, \partial b_{i}(r) / \partial r_{j}, d \phi / d t$ and constants $a_{0}, a_{1} ;$ all the derivatives are generalized.

Proof. From Lemma 5, we take $\tau \in\left(0, \tau_{0}\right)$. There are subsequences of $\left\{x^{(n)}(\theta, t)\right\}$ and $\left\{r^{(n)}(x, t)\right\}$, which converge uniformly, the derivatives of which weakly converge in $L_{2}$ for $t \in\left[\tau, \tau_{0}\right]$; the limiting functions are Lipschitz continuous. By the diagonal subsequence technique, we get the subsequences which have the above convergence as $t \in\left[\tau, \tau_{0}\right]$ and $\tau>0$ is arbitrary. It is obvious that the limiting functions are the solutions of this problem and satisfy the inequalities; as for (4.35), it can be obtained by (2.1) and (4.33). The function $p$ has a positive lower bound at $t=0$, inequality $|\partial R / \partial t| \leqslant M_{1}$ implies that (4.34) holds on $t \in$ $\left[0, \tau_{0}^{\prime}\right]$ for a suitable $\tau_{0}^{\prime}$, if $\tau_{0}^{\prime}<\tau_{0}$; we can take $\tau_{0}^{\prime}$ for $\tau_{0}$. Q.E.D. 
The above discussion is concerned with the forward wave; it is all the same for backward waves.

5. One case of the discontinuous initial value problem. We are going to discuss the problem (1.1), (1.5) given in the introduction. Suppose

(5.1) functions $\rho_{1,0}\left(x^{\prime}\right), \rho_{2,0}\left(x^{\prime}\right), u_{0}\left(x^{\prime}\right), p_{0}\left(x^{\prime}\right)$ satisfy the Lipschitz condition on the intervals $(-\infty, 0)$ and $(0,+\infty)$,

(5.2) $p_{0}\left(x^{\prime}\right) \geqslant \delta>0$,

(5.3) $\rho_{1,0}\left(x^{\prime}\right) \geqslant 0, \rho_{2,0}\left(x^{\prime}\right) \geqslant 0, \rho_{1,0}\left(x^{\prime}\right)+\rho_{2,0}\left(x^{\prime}\right) \geqslant \delta>0$.

Let

$$
\begin{aligned}
& \lim _{x^{\prime} \rightarrow 0+}\left(\rho_{1,0}\left(x^{\prime}\right), \rho_{2,0}\left(x^{\prime}\right), u_{0}\left(x^{\prime}\right), p_{0}\left(x^{\prime}\right)\right)=\left(\rho_{1 r}, \rho_{2 r}, u_{r}, p_{r}\right), \\
& \lim _{x^{\prime} \rightarrow 0-}\left(\rho_{1,0}\left(x^{\prime}\right), \rho_{2,0}\left(x^{\prime}\right), u_{0}\left(x^{\prime}\right), p_{0}\left(x^{\prime}\right)\right)=\left(\rho_{1 l}, p_{2 l}, u_{l}, p_{l}\right) .
\end{aligned}
$$

Definition 1. If (5.4) holds, then (1.6)-(1.7) is called the corresponding problem of (1.1)-(1.5).

We have proved the existence of the solutions of problem (1.6)-(1.7). Now we discuss the solutions of problem (1.1)-(1.5), the structure of which near the origin is:

(a) There is a double characteristic contact discontinuous at the $t$-axis, namely, $u$ and $p$ are continuous, but $\rho_{1}$ and $\rho_{2}$ are discontinuous in general.

(b) There is a forward wave starting from the origin in the first quadrant which is either a shock wave or a rarefaction wave.

(c) There is also a backward shock wave or a backward rarefaction wave in the second quadrant.

(d) At the four regions divided by these three waves the solutions satisfy the Lipschitz condition.

DEFINITION 2. If problem (1.6)-(1.7) is the corresponding problem of (1.1)-(1.5), the solutions defined by (5.5) and Theorem 1 exist, and if the following conditions are satisfied, then it is said that the solutions have the identical structure near the origin.

(a) Each shock wave corresponds to a shock wave, each rarefaction wave corresponds to a rarefaction wave.

(b) The slopes of the shock waves at the origin are equal.

(c) For rarefaction waves the correspondence of (4.1), (4.2) and (2.12), (4.3) holds.

(d) Within the regions which the solutions are Lipschitz continuous the limits of the solutions are equal as $x \rightarrow 0, t \rightarrow 0$.

Problem (1.6)-(1.7) is equivalent to problem (3.1)-(3.2). We use the symbols of Theorem 1 in the following, say, $\bar{u}, \bar{p}$, and so on. We are aiming at proving the following theorem in this section. 
TheOREM 3. If $p_{l}>\bar{p}>p_{r}$, then the solutions of problem (1.1), (1.5) exist on $t \in\left[0, \tau_{0}\right],-\infty<x<+\infty$, and have the identical structure near the origin to that of problem (1.6), (1.7), where $\tau_{0}>0$ depends only on the upper and lower bounds of the initial data (1.5) and their Lipschitz coefficients.

We divide the proof of this theorem into two parts.

Part 1. First: From [9], we obtain the Lipschitz continuous solutions on domains $\left\{(x, t) \mid t \in\left[0, \tau_{0}\right],-\infty<x \leqslant x_{0}(t)\right\}$ and $\left\{(x, t) \mid t \in\left[0, \tau_{0}\right], x_{1}(t)<x<+\infty\right\}$, where $x_{0}(t), x_{1}(t)$ are characteristic curves

$$
d x / d t=\lambda_{1}(r(x, t)),\left.\quad x\right|_{t=0}=0,
$$

and

$$
d x / d t=\lambda_{4}(r(x, t)),\left.\quad x\right|_{t=0}=0 .
$$

From Theorem 1, there is a backward rarefaction wave and a forward shock for problem (3.1), (3.2). From Theorem 2, we have the existence of the backward rarefaction wave of problem (1.1), (1.5) which exists on the domain $t \in\left(0, \tau_{0}\right]$, $x_{0}(t) \leqslant x \leqslant x_{l}(t)$, where $x_{l}(t)$ is another characteristic curve satisfying (5.6), and we know from Theorem 2 that

$$
\begin{array}{ll}
\lim _{t \rightarrow 0} p\left(x_{l}(t), t\right)=\bar{p}, & \lim _{t \rightarrow 0} u\left(x_{l}(t), t\right)=\bar{u}, \\
\lim _{t \rightarrow 0} v\left(x_{l}(t), t\right)=\hat{v}, & \lim _{t \rightarrow 0} c\left(x_{l}(t), t\right)=\hat{c} .
\end{array}
$$

The remaining problem is to prove the existence of the shock wave $x=x_{r}(t)$ which satisfies $x_{r}(t) \geqslant x_{1}(t), x_{r}(0)=0$, and there exist Lipschitz continuous solutions on $\left\{(x, t) \mid t \in\left[0, \tau_{0}\right], x_{l}(t) \leqslant x<0\right\}$ and $\left\{(x, t) \mid t \in\left[0, \tau_{0}\right], 0<x<x_{r}(t)\right\}$ which are continuous at $x=x_{l}(t)$ and satisfy (2.13)-(2.16) at $x=x_{r}(t)$. Because $x_{r}(t)$ is unknown, this is a free boundary problem. Now we extricate ourselves from this problem temporarily and consider a fixed boundary problem.

We discuss system (2.7) and the following conditions.

$$
\begin{gathered}
w\left(x_{l}(t), t\right)=\left[\begin{array}{c}
u \\
p \\
c \\
s
\end{array}\right]_{x=x_{r}(t)}=\phi_{l}(t) \\
\left.\frac{u-u_{r}(t)}{p-p_{r}(t)}\right|_{x=x_{r}(t)}=-\left.\left\{\frac{2 v_{r}(t)}{\left(\gamma_{c, r}(t)+1\right) p+\left(\gamma_{c, r}(t)-1\right) p_{r}(t)}\right\}^{1 / 2}\right|_{x=x_{r}(t)} \\
\left.c\right|_{x=x_{r}(t)}=c_{r}(t) \\
v /\left.v_{r}(t)\right|_{x=x_{r}(t)}=\left.\frac{\left(\gamma_{c, r}(t)-1\right) p+\left(\gamma_{c, r}(t)+1\right) p_{r}(t)}{\left(\gamma_{c, r}(t)+1\right) p+\left(\gamma_{c, r}(t)-1\right) p_{r}(t)}\right|_{x=x_{r}(t)} \\
\bar{l}_{1}\left(\phi_{l}(t)\right) \phi_{l}^{\prime}(t)=b_{1}\left(\phi_{l}(t)\right), \quad x_{l}^{\prime}(t)=\lambda_{1}\left(\phi_{l}(t)\right) \\
\phi_{l}(0)=\left[\begin{array}{c}
\bar{u} \\
\bar{p} \\
\hat{c} \\
s(\hat{v}, \bar{p}, \hat{c})
\end{array}\right)
\end{gathered}
$$




$$
\begin{aligned}
& u(0+, t)=u(0-, t), \quad p(0+, t)=p(0-, t), \\
& x_{r}^{\prime}(0)=\left\{\frac{\left(\gamma_{c, r}(0)+1\right) \bar{p}+\left(\gamma_{c, r}(0)-1\right) p_{r}(0)}{2 v_{r}(0)}\right\}^{1 / 2},
\end{aligned}
$$

where $x_{l}(t), x_{r}(t) \in C^{1}, x_{r}^{\prime}(t) \leqslant \bar{\lambda}_{4}-\varepsilon, \varepsilon>0, \bar{\lambda}_{4}=\lambda_{4}(\bar{p}, \bar{c}, s(\bar{v}, \bar{p}, \bar{c}))$, and $v_{r}(t)$, $u_{r}(t), p_{r}(t), \gamma_{c, r}(t), c_{r}(t)$ are Lipschitz continuous functions equal to the value of the solutions at the curve $x=x_{r}(t)$.

Let the functions $u, p, c, s$ be solutions of (2.7), (5.8)-(5.15); we derive some integral relations at first.

Let $\xi=\xi(\eta, x, t)$ be a characteristic curve passing through the point $(x, t)$, satisfying

$$
d \xi / d \eta=\lambda_{i}(w(\xi, \eta)),\left.\quad \xi\right|_{\eta=t}=x
$$

Let $(\xi, \tau)$ be the intersection point of $\xi=\xi(\eta, x, t)$ with boundary curves $\xi=$ $x_{l}(\eta), \xi=x_{r}(\eta)$ or contact discontinuous at $\xi=0$ and $\tau<t$. We integrate every equation in (2.7) along the corresponding characteristic curves, respectively, and obtain

$$
\begin{gathered}
u+\left.l_{12}(p, c, s) p\right|_{\tau} ^{t}=\int_{\tau}^{t} p \frac{\partial l_{12}}{\partial \eta} d \eta \\
\left.c\right|_{\tau} ^{t}=\int_{\tau}^{t} f d \eta \\
\left.S\right|_{\tau} ^{t}=\int_{\tau}^{t} h d \eta \\
u+\left.l_{42}(p, c, s) p\right|_{\tau} ^{t}=\int_{\tau}^{t} p \frac{\partial l_{42}}{\partial \eta} d \eta .
\end{gathered}
$$

We solve (2.7), (5.8)-(5.15) by iteration. First take smooth functions $v_{r}^{(n)}(t)$, $u_{r}^{(n)}(t), \ldots$ which converge uniformly to functions $v_{r}(t), u_{r}(t), \ldots$ and are bounded uniformly in the space $C^{1}$, and $v_{r}^{(n)}(0)=v_{r}(0), u_{r}^{(n)}(0)=u_{r}(0), \ldots$ We introduce $w^{(0)}(x, t)$ as the 0 th approximation which is taken as

$$
(v, c, u, p)= \begin{cases}(\bar{v}, \bar{c}, \bar{u}, \bar{p}) & \text { for } x>0 \\ (\hat{v}, \hat{c}, \bar{u}, \bar{p}) & \text { for } x<0\end{cases}
$$

If $w^{(n-1)}(x, t)$ is known, we determine $w^{(n)}(x, t)$. First of all, we determine the functions $u^{(n)}+l_{42}^{+} p^{(n)}$ and $u^{(n)}+l_{12}^{-} p^{(n)}$ at contact discontinuities, where the symbols " + " and " -" denote the right and left limit, respectively, if the independent variables are written; those are

$$
\begin{aligned}
& l_{42}^{+}=l_{42}\left(p^{(n-1)}(0, t), c^{(n-1)}(0+, t), s^{(n-1)}(0+, t)\right), \\
& l_{12}^{-}=l_{12}\left(p^{(n-1)}(0, t), c^{(n-1)}(0-, t), s^{(n-1)}(0-, t)\right) .
\end{aligned}
$$


For simplification we do not write the independent variables later. It is obvious that

$$
\begin{aligned}
& u^{(n)}+l_{42}^{+} p^{(n)}=\frac{l_{42}^{+}-l_{12}^{+}}{l_{42}^{-}-l_{12}^{+}}\left(u^{(n)}+l_{42}^{-} p^{(n)}\right)+\frac{l_{42}^{-}-l_{42}^{+}}{l_{42}^{-}-l_{12}^{+}}\left(u^{(n)}+l_{12}^{+} p^{(n)}\right), \\
& u^{(n)}+l_{12}^{-} p^{(n)}=\frac{l_{12}^{-}-l_{42}^{-}}{l_{12}^{+}-l_{42}^{-}}\left(u^{(n)}+l_{12}^{+} p^{(n)}\right)+\frac{l_{12}^{+}-l_{12}^{-}}{l_{12}^{+}-l_{42}^{-}}\left(u^{(n)}+l_{42}^{-} p^{(n)}\right) .
\end{aligned}
$$

$u^{(n)}+l_{42}^{-} p^{(n)}$ and $u^{(n)}+l_{12}^{+} p^{(n)}$ are given, referring to (5.17) and (5.20), as

$$
\begin{aligned}
& u^{(n)}+l_{42}^{-} p^{(n)}=u^{(n-1)}+\left.l_{42}^{(n-1)} p^{(n-1)}\right|_{\tau}+\int_{\tau}^{t} p^{(n-1)} \frac{\partial l_{42}^{(n-1)}}{\partial \eta} d \eta, \\
& u^{(n)}+l_{12}^{+} p^{(n)}=u^{(n-1)}+\left.l_{12}^{(n-1)} p^{(n-1)}\right|_{\tau}+\int_{\tau}^{t} p^{(n-1)} \frac{\partial l_{12}^{(n-1)}}{\partial \eta} d \eta,
\end{aligned}
$$

where the two $\tau$ 's are not, in general, equal and the integrations are taken along different characteristic curves; we do not distinguish them because it cannot cause any misunderstanding. Besides,

$$
l_{42}^{(n-1)}=l_{42}\left(p^{(n-1)}, c^{(n-1)}, s^{(n-1)}\right) .
$$

We consider the system

$\left(d / d t_{i}\right)\left[\bar{l}_{i}\left(w^{(n-1)}\right) w^{(n)}\right]=b_{i}\left(w^{(n-1)}\right)+\left[d \bar{l}_{i}\left(w^{(n-1)}\right) / d t_{i}\right] w^{(n-1)}, \quad i=1,2,3,4$,

and solve it in the first quadrant and the second quadrant, respectively, under conditions (5.8)-(5.11) and the assumption that $u^{(n)}+\left.l_{42}^{+} p^{(n)}\right|_{x=0}, u^{(n)}+\left.l_{12}^{-} p^{(n)}\right|_{x=0}$ are known. System (5.23) is linear; the problem in the second quadrant is a Goursat problem and the existence of the solutions is well known. The existence in the first quadrant is also easy to prove; we only have to point out that if $(x, t)$ lies on the right boundary, similarly to (5.20), we have

$$
u^{(n)}+\left.l_{42}^{(n)} p^{(n)}\right|_{(x, t)}=u^{(n)}+\left.l_{42}^{+} p^{(n)}\right|_{(0, t)}+\int_{\tau}^{t} p^{(n-1)} \frac{\partial l_{42}^{(n-1)}}{\partial \eta} d \eta
$$

Combining (5.24) with (5.9) we can determine $u^{(n)}$ and $p^{(n)}$ uniquely. From (5.10) and (5.11), $c^{(n)}$ and $v^{(n)}$ can be determined uniquely, and then $s^{(n)}$.

From now on $K$ denotes a constant which depends only on the upper and lower bounds of the initial data (1.5) and their Lipschitz cofficients.

Lemma 6. Constants $\tau_{0}>0, M_{0}, M_{1}$, which depend only on constants $K$ and $\varepsilon$, exist such that

$$
\left|w^{(n)}(x, t)\right| \leqslant M_{0}, \quad\left|\partial w^{(n)} / \partial x\right|,\left|\partial w^{(n)} / \partial t\right| \leqslant M_{1},
$$

for $t \in\left[0, \tau_{0}\right], x_{l}(t) \leqslant x \leqslant x_{r}(t)$.

Proof. We use induction; if the above result holds for $n-1$, we estimate $w^{(n)}$. Similarly, for the proof of Lemma 5 , for each solution the above estimation holds 
on a certain neighborhood of the origin, hence

$$
\begin{array}{ll}
\left|w^{(n)}(x, t)-w^{(0)}(0+, 0)\right|<K M_{1} t & \text { for } x>0, \\
\left|w^{(n)}(x, t)-w^{(0)}(0-, 0)\right|<K M_{1} t & \text { for } x<0 .
\end{array}
$$

Now we estimate the first order derivatives. First of all, we estimate the values of derivatives at the curve $x=x_{r}(t)$. Take one point $(0, \tau)$ at the $t$-axis arbitrarily, draw the characteristic curves of the first and fourth families passing through this point; these curves intersect the boundary curves at $\left(\xi_{1}, \tau_{1}\right),\left(\xi_{2}, \tau_{2}\right),\left(\xi_{3}, \tau_{3}\right)$ (see Figure 1).

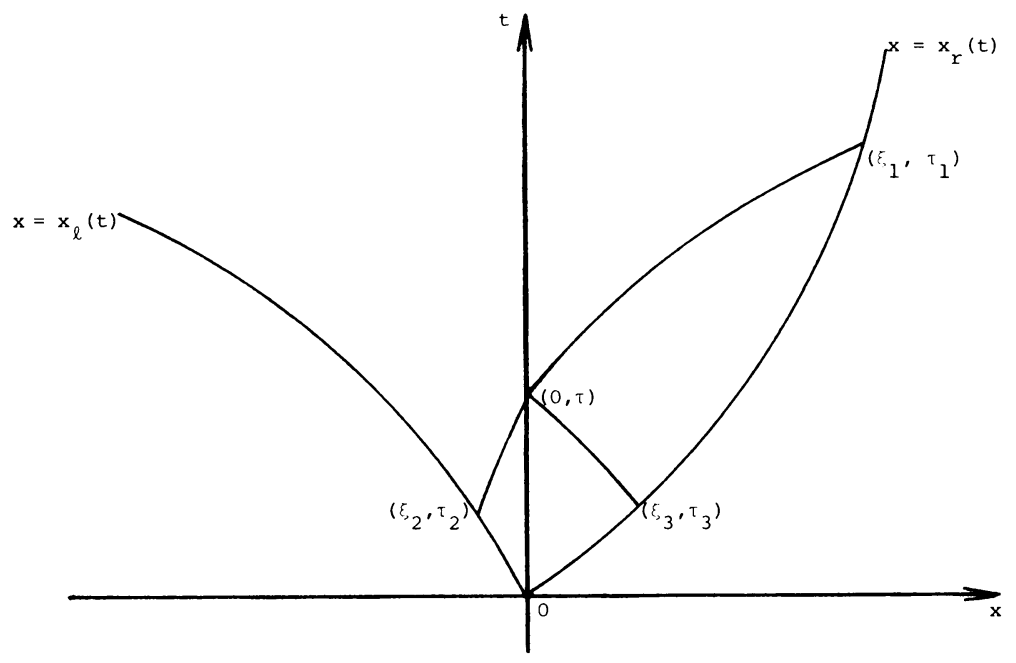

Figure 1

Let the inverse function of $\xi=\xi(\eta, x, t)$ be $\eta=\eta(\xi, x, t)$, which satisfies

$$
\begin{aligned}
& d \eta / d \xi=1 / \lambda_{i}\left(w^{(n-1)}(\xi, \eta)\right),\left.\quad \eta\right|_{\xi=x}=t \\
& \frac{\partial u^{(n)}}{\partial t}+l_{42}^{-}\left.\frac{\partial p^{(n)}}{\partial t}\right|_{(0, \tau)}=-\left.\frac{\partial l_{42}^{-}}{\partial t} p^{(n)}\right|_{(0, \tau)} \\
&+\left(\frac{d u^{(n-1)}}{d \tau_{2}}+l_{42}^{(n-1)} \frac{d p^{(n-1)}}{d \tau_{2}}+\frac{d l_{42}^{(n-1)}}{d \tau_{2}} p^{(n-1)}\right)_{\left(\xi_{2}, \tau_{2}\right)} \frac{d \tau_{2}}{d t} \\
&-\left(p^{(n-1)} \frac{\partial l_{42}^{(n-1)}}{\partial \xi}\right)_{\left(\xi_{2}, \tau_{2}\right)} \frac{d \xi_{2}}{d t} \\
&+\left.\left(\frac{\partial l_{42}^{(n-1)}}{\partial t} p^{(n-1)}\right)\right|_{\xi_{2}} ^{0}+\int_{\xi_{2}}^{0}\left[\frac{\partial p^{(n-1)}}{\partial t} \frac{\partial l_{42}^{(n-1)}}{\partial \xi}-\frac{\partial l_{42}^{(n-1)}}{\partial t} \frac{\partial p^{(n-1)}}{\partial \xi}\right] d \xi
\end{aligned}
$$

where $\tau_{2}$ is determined by

$$
\xi_{2}=x_{l}\left(\tau_{2}\right), \quad \tau_{2}=\eta\left(\xi_{2}, 0, t\right)
$$

hence

$$
\frac{d \tau_{2}}{d t}=\frac{\lambda_{4}(\partial \eta / \partial t)}{\lambda_{4}-x_{l}^{\prime}}
$$


From (5.27),

$$
\frac{\partial \eta}{\partial t}=\exp \left\{\int_{x}^{\xi} \frac{\partial}{\partial \eta}\left(\frac{1}{\lambda_{4}}\right) d \xi\right\}
$$

On the other hand,

$$
\frac{d l_{22}^{(n-1)}}{d \tau_{2}} \frac{d \tau_{2}}{d t}=\frac{d l_{42}^{(n-1)}}{d t}=\frac{\partial l_{42}^{(n-1)}}{\partial t}+\frac{d \xi_{2}}{d t} \frac{\partial l_{42}^{(n-1)}}{\partial \xi}
$$

therefore

$$
\begin{aligned}
\frac{\partial u^{(n)}}{\partial t}+\left.l_{42}^{-} \frac{\partial p^{(n)}}{\partial t}\right|_{(0, \tau)}= & \left(\frac{d u^{(n-1)}}{d \tau_{2}}+l_{42}^{(n-1)} \frac{d p^{(n-1)}}{d \tau_{2}}\right)_{\left(\xi_{2}, \tau_{2}\right)} \frac{\lambda_{4}}{\lambda_{4}-x_{l}^{\prime}} \\
& \cdot \exp \left\{\int_{0}^{\xi_{2}} \frac{\partial}{\partial \eta}\left(\frac{1}{\lambda_{4}}\right) d \xi\right\}+\left.\frac{\partial l_{42}^{-}}{\partial t}\left(p^{(n-1)}-p^{(n)}\right)\right|_{(0, \tau)} \\
& +\int_{\xi_{2}}^{0}\left[\frac{\partial p^{(n-1)}}{\partial t} \frac{\partial l_{42}^{(n-1)}}{\partial \xi}-\frac{\partial l_{42}^{(n-1)}}{\partial t} \frac{\partial p^{(n-1)}}{\partial \xi}\right] d \xi
\end{aligned}
$$

Similarly,

$$
\begin{aligned}
\frac{\partial u^{(n)}}{\partial t}+\left.l_{12}^{+} \frac{\partial p^{(n)}}{\partial t}\right|_{(0, \tau)}= & \left.\left(\frac{d u^{(n-1)}}{d \tau_{3}}+l_{12}^{(n-1)} \frac{d p^{(n-1)}}{d \tau_{3}}\right)\right|_{\left(\xi_{3}, \tau_{3}\right)} \frac{-\lambda_{1}}{-\lambda_{1}+x_{r}^{\prime}} \\
& \cdot \exp \left\{\int_{0}^{\xi_{3}} \frac{\partial}{\partial \eta}\left(\frac{1}{\lambda_{1}}\right) d \xi\right\}+\left.\frac{\partial l_{12}^{+}}{\partial t}\left(p^{(n-1)}-p^{(n)}\right)\right|_{(0, \tau)} \\
& +\int_{\xi_{3}}^{0}\left[\frac{\partial p^{(n-1)}}{\partial t} \frac{\partial l_{12}^{(n-1)}}{\partial \xi}-\frac{\partial l_{12}^{(n-1)}}{\partial t} \frac{\partial p^{(n-1)}}{\partial \xi}\right] d \xi
\end{aligned}
$$

From (5.23),

$$
u^{(n)}+\left.l_{42}^{(n-1)} p^{(n)}\right|_{\left(\xi_{1}, \tau_{1}\right)}=u^{(n)}+\left.l_{42}^{+} p^{(n)}\right|_{(0, \tau)}+\int_{0}^{\xi_{1}} p^{(n-1)} \frac{\partial l_{42}^{(n-1)}}{\partial \xi} d \xi
$$

Differentiate this with respect to $\tau_{1}$ and obtain

$$
\begin{aligned}
\frac{\partial u^{(n)}}{\partial \tau_{1}}+l_{42}^{(n-1)} & \left.\frac{\partial p^{(n)}}{\partial \tau_{1}}\right|_{\left(\xi_{1}, \tau_{1}\right)} \\
= & \left.\left(\frac{\partial u^{(n)}}{\partial t}+l_{42}^{+} \frac{\partial p^{(n)}}{\partial t}\right) \frac{\partial \eta}{\partial t}\right|_{(0, \tau)}+\left.\frac{\partial l_{42}^{(n-1)}}{\partial \tau_{1}}\left(p^{(n-1)}-p^{(n)}\right)\right|_{0} ^{\xi_{1}} \\
& +\int_{0}^{\xi_{1}}\left[\frac{\partial p^{(n-1)}}{\partial \tau_{1}} \frac{\partial l_{42}^{(n-1)}}{\partial \xi}-\frac{\partial p^{(n-1)}}{\partial \xi} \frac{\partial l_{42}^{(n-1)}}{\partial \tau_{1}}\right] d \xi
\end{aligned}
$$


From (5.23) and (5.28),

$$
\begin{aligned}
\frac{d u^{(n)}}{d \tau_{1}}+\left.l_{42}^{(n-1)} \frac{d p^{(n)}}{d \tau_{1}}\right|_{\left(\xi_{1}, \tau_{1}\right)} & \left(\frac{\partial u^{(n)}}{\partial \tau_{1}}+x_{r}^{\prime} \frac{\partial u^{(n)}}{\partial \xi_{1}}\right)+\left.l_{42}^{(n-1)}\left(\frac{\partial p^{(n)}}{\partial \tau_{1}}+x_{r}^{\prime} \frac{\partial p^{(n)}}{\partial \xi_{1}}\right)\right|_{\left(\xi_{1}, \tau_{1}\right)} \\
= & \frac{x_{r}^{\prime}}{\lambda_{4}}\left[\left(\frac{\lambda_{4}}{x_{r}^{\prime}} \frac{\partial u^{(n)}}{\partial \tau_{1}}+\lambda_{4} \frac{\partial u^{(n)}}{\partial \xi_{1}}\right)+l_{42}^{(n-1)}\left(\frac{\lambda_{4}}{x_{r}^{\prime}} \frac{\partial p^{(n)}}{\partial \tau_{1}}+\lambda_{4} \frac{\partial p^{(n)}}{\partial \xi_{1}}\right)\right]_{\left(\xi_{1}, \tau_{1}\right)} \\
= & \frac{\lambda_{4}-x_{r}^{\prime}}{\lambda_{4}}\left\{\left(\frac{\partial u^{(n)}}{\partial t}+l_{42}^{+} \frac{\partial p^{(n)}}{\partial t}\right) \exp \left\{\int_{0}^{\xi_{1}} \frac{\partial}{\partial \eta}\left(\frac{1}{\lambda_{4}}\right) d \xi\right\}\right. \\
& +\int_{0}^{\xi_{1}}\left[\frac{\partial p^{(n-1)}}{\partial \tau_{1}} \frac{\partial l_{42}^{(n-1)}}{\partial \xi}+\frac{\partial p^{(n-1)}}{\partial \xi} \frac{\partial l_{42}^{(n-1)}}{\partial \tau_{1}}\right] d \xi \\
& \left.+\left.\frac{x_{r}^{\prime}}{\lambda_{4}}\left(p^{(n-1)}-p^{(n)}\right)\left(\frac{2 l_{42}^{(n-1)}}{\partial \tau_{1}}+\lambda_{4}^{(n-1)} \frac{\partial l_{42}^{(n-1)}}{\partial \xi_{1}}\right)_{\left(\xi_{1}, \tau_{1}\right)}^{\partial \tau_{1}}\left(p^{(n-1)}-p^{(n)}\right)\right|_{0} ^{\xi_{1}}\right\}
\end{aligned}
$$

From (5.26),

$$
\begin{aligned}
\left|p^{(n-1)}(x, t)-p^{(n)}(x, t)\right| \leqslant & \left|p^{(n-1)}(x, t)-p^{(n-1)}(0,0)\right| \\
& +\left|p^{(n)}(x, t)-p^{(n)}(0,0)\right| \leqslant K M_{1} t .
\end{aligned}
$$

From (5.25), expressions (5.29)-(5.31) can be written in the following concise form:

$$
\begin{aligned}
& \frac{\partial u^{(n)}}{\partial t}+\left.l_{42}^{-} \frac{\partial p^{(n)}}{\partial t}\right|_{(0, \tau)}= \frac{\lambda_{4}}{\lambda_{4}-x_{l}^{\prime}} \exp \left\{\int_{0}^{\xi_{2}} \frac{\partial}{\partial \eta}\left(\frac{1}{\lambda_{4}}\right) d \xi\right\} \\
& \cdot\left(\frac{d u^{(n-1)}}{d \tau_{2}}+l_{42}^{(n-1)} \frac{d p^{(n-1)}}{d \tau_{2}}\right)_{\left(\xi_{2}, \tau_{2}\right)}+R_{1} \\
& \frac{\partial u_{n}}{\partial t}+\left.l_{12}^{+} \frac{\partial p^{(n)}}{\partial t}\right|_{(0, \tau)}= \frac{\lambda_{1}}{\lambda_{1}-x_{r}^{\prime}} \exp \left\{\int_{0}^{\xi_{3}} \frac{\partial}{\partial \eta}\left(\frac{1}{\lambda_{1}}\right) d \xi\right\} \\
& \cdot\left(\frac{d u^{(n-1)}}{d \tau_{3}}+l_{12}^{(n-1)} \frac{d p^{(n-1)}}{d \tau_{3}}\right)_{\left(\xi_{3}, \tau_{3}\right)}+R_{2} \\
& \frac{d u^{(n)}}{d \tau_{1}}+\left.l_{42}^{(n-1)} \frac{d p^{(n)}}{d \tau_{1}^{\prime}}\right|_{\left(\xi_{1}, \tau_{1}\right)}=\frac{\lambda_{4}-x_{r}^{\prime}}{\lambda_{4}} \exp \left\{\int_{0}^{\xi_{1}} \frac{\partial}{\partial \eta}\left(\frac{1}{\lambda_{4}}\right) d \xi\right\} \\
& \cdot\left(\frac{\partial u^{(n)}}{\partial t}+l_{42}^{+} \frac{\partial p^{(n)}}{\partial t}\right)_{(0, \tau)}+R_{3}
\end{aligned}
$$

where $\left|R_{1}\right|,\left|R_{2}\right|,\left|R_{3}\right| \leqslant K M_{1}^{2} \tau_{1}$. 
We note that (5.21) still holds if we change $\partial u^{(n)} / \partial t$ and $\partial p^{(n)} / \partial t$ for $u^{(n)}$ and $p^{(n)}$; hence

$$
\begin{gathered}
\left(\frac{d u^{(n)}}{d \tau_{1}}+l_{42}^{(n-1)} \frac{d p^{(n)}}{d \tau_{1}}\right)_{\left(\xi_{1}, \tau_{1}\right)}=\frac{\lambda_{4}-x_{r}^{\prime}}{\lambda_{4}} \exp \left\{\int_{0}^{\xi_{1}} \frac{\partial}{\partial \eta}\left(\frac{1}{\lambda_{4}}\right) d \xi\right\} \\
\cdot\left\{\frac { l _ { 4 2 } ^ { + } - l _ { 1 2 } ^ { + } } { l _ { 4 2 } ^ { - } - l _ { 1 2 } ^ { + } } \left[\frac{\lambda_{4}}{\lambda_{4}-x_{l}^{\prime}} \exp \left\{\int_{0}^{\xi_{2}} \frac{\partial}{\partial \eta}\left(\frac{1}{\lambda_{4}}\right) d \xi\right\}\right.\right. \\
\left.\cdot\left(\frac{d u^{(n-1)}}{d \tau_{2}}+l_{42}^{(n-1)} \frac{d p^{(n-1)}}{d \tau_{2}}\right)_{\left(\xi_{2}, \tau_{2}\right)}\right] \\
+\frac{l_{42}^{-}-l_{42}^{+}}{l_{42}^{-}-l_{12}^{+}}\left[\frac{\lambda_{1}}{\lambda_{1}-x_{r}^{\prime}} \exp \left\{\int_{0}^{\xi_{2}} \frac{\partial}{\partial \eta}\left(\frac{1}{\lambda_{1}}\right) d \xi\right\}\right. \\
\left.\left.\cdot\left(\frac{d u^{(n-1)}}{d \tau_{3}}+l_{12}^{(n-1)} \frac{d p^{(n-1)}}{d \tau_{3}}\right)_{\left(\xi_{3}, \tau_{3}\right)}\right]\right\}+R,
\end{gathered}
$$

where $|R| \leqslant K M_{1}^{2} \tau_{1}$. We note that

$$
\begin{gathered}
\left|\frac{\lambda_{4}-x_{r}^{\prime}}{\lambda_{4}}\right|,\left|\frac{\lambda_{4}}{\lambda_{4}-x_{l}^{\prime}}\right|,\left|\frac{\lambda_{1}}{\lambda_{1}-x_{r}^{\prime}}\right|<1, \\
\exp \left\{\int_{0}^{\xi_{1}} \frac{\partial}{\partial \eta}\left(\frac{1}{\lambda_{4}}\right) d \xi\right\}, \exp \left\{\int_{0}^{\xi_{2}} \frac{\partial}{\partial \eta}\left(\frac{1}{\lambda_{4}}\right) d \xi\right\}, \\
\exp \left\{\int_{0}^{\xi_{3}} \frac{\partial}{\partial \eta}\left(\frac{1}{\lambda_{1}}\right) d x\right\} \leqslant \exp \left\{K M_{1} \tau_{1}\right\}, \\
\left(\frac{d u^{(n-1)}}{d \tau_{2}}+l_{42}^{(n-1)} \frac{d p^{(n-1)}}{d \tau_{2}}\right)_{\left(\xi_{2}, \tau_{2}\right)}=\left.\bar{l}_{4}\left(\phi_{l}(t)\right) \phi_{l}^{\prime}(t)\right|_{\left(\xi_{2}, \tau_{2}\right)}, \\
\left|\frac{l_{42}^{-}-l_{42}^{+}}{l_{42}^{-}-l_{12}^{+}}\right|=\left|\frac{l_{42}^{-}-l_{42}^{+}}{l_{42}^{-}+l_{42}^{+}}\right| \leqslant 1 .
\end{gathered}
$$

Therefore,

$$
\begin{aligned}
& \left|\left(\frac{d u^{(n)}}{d \tau_{1}}+l_{42}^{(n-1)} \frac{d p^{(n)}}{d \tau_{1}}\right)_{\left(\xi_{1}, \tau_{1}\right)}\right| \\
& \leqslant e^{K M_{1} \tau_{1}}\left|\left(\frac{d u^{(n-1)}}{d \tau_{3}}+l_{12}^{(n-1)} \frac{d p^{(n-1)}}{d \tau_{3}}\right)_{\left(\xi_{3}, \tau_{3}\right)}\right|+K+K M_{1}^{2} \tau_{1} .
\end{aligned}
$$

From Lemma 1,

$$
\begin{aligned}
\left(d u^{(n-1)} / d \tau_{3}+\right. & \left.l_{12}^{(n-1)}\left(d p^{(n-1)} / d \tau_{3}\right)\right)_{\left(\xi_{3}, \tau_{3}\right)} \\
& =\beta\left(d u^{(n-1)} / d \tau_{3}+l_{42}^{(n-1)}\left(d p^{(n-1)} / d \tau_{3}\right)\right)+G\left(p^{(n-1)}, \tau_{3}\right)
\end{aligned}
$$

hence

$$
\begin{aligned}
& \left|\left(d u^{(n)} / d \tau_{1}+l_{42}^{(n-1)}\left(d p^{(n)} / d \tau_{1}\right)\right)_{\left(\xi_{1}, \tau_{1}\right)}\right| \\
& \leqslant \beta e^{K M_{1} \tau_{1}}\left|\left(d u^{(n-1)} / d \tau_{3}+l_{42}^{(n-1)}\left(d p^{(n-1)} / d \tau_{3}\right)\right)_{\left(\xi_{3}, \tau_{3}\right)}\right|+K+K M_{1}^{2} \tau_{1} .
\end{aligned}
$$


(5.26) implies $\left|l_{42}^{(n-1)}-l_{42}^{(n-2)}\right| \leqslant K M_{1} t$; hence

$$
\begin{aligned}
\mid\left(d u^{(n)} / d \tau_{1}\right. & \left.+l_{42}^{(n-1)}\left(d p^{(n)} / d \tau_{1}\right)\right)_{\left(\xi_{1}, \tau_{1}\right)} \mid \\
& \leqslant \beta e^{K M_{1} \tau_{1}}\left|\left(\frac{d u^{(n-1)}}{d \tau_{3}}+l_{42}^{(n-2)} \frac{d p^{(n-1)}}{d \tau_{3}}\right)_{\left(\xi_{3}, \tau_{3}\right)}\right|+K+K M_{1}^{2} \tau_{1} .
\end{aligned}
$$

For $t \in\left[0, \tau_{0}\right]$ we define on the curve $x=x_{r}(t)$ that

$$
g^{(n)}(t)=d u^{(n)} / d t+l_{42}^{(n-1)}\left(d p^{(n)} / d t\right)
$$

Let $U=\max _{t, n}\left|g^{(n)}(t)\right|$; then

$$
U \leqslant \beta e^{K M_{1} \tau_{0}} U+K+K M_{1}^{2} \tau_{0} .
$$

The bound of $\beta$ is less than 1 on a bounded region; we take $\tau_{0}$ such that $\beta e^{K M_{1} t} \leqslant 1-\delta, \delta>0$ for $t \in\left[0, \tau_{0}\right]$; then

$$
U \leqslant \delta^{-1}\left[K+K M_{1}^{2} \tau_{0}\right]
$$

From (5.34) and (5.9)-(5.11), we have

$$
\left|(d / d t) w^{(n)}\left(x_{r}(t), t\right)\right| \leqslant(K / \delta)\left(1+M_{1}^{2} \tau_{0}\right) .
$$

Applying (5.35) we can estimate $\partial w^{(n)} / \partial x, \partial w^{(n)} / \partial t$ similar to the proof in Lemma 5; then we use the estimation and (5.26) to obtain $\tau_{0}, M_{0}, M_{1}$; therefore the result of this lemma holds. The above technique is similar to that of Lemma 5 , so we omit it. We only point out that in order to assure that the characteristic curves of the fourth family intersect the curve $x=x_{r}(t)$ at one point $\left(\xi_{1}, \tau_{1}\right)$, we demand that $x_{r}^{\prime} \leqslant \lambda_{4}\left(w^{(n)}(x, t)\right)-\varepsilon / 2$ always holds; but

$$
\left|\lambda_{4}\left(w^{(n)}(x, t)\right)-\bar{\lambda}_{4}\right| \leqslant K M_{1} t
$$

so we take the constant $\tau_{0}$ such that $K M_{1} \tau_{0} \leqslant \varepsilon / 2$. Q.E.D.

LemMa 7. There exist constants $\tau_{0}>0, M_{0}, M_{1}$ which depend only on $K$ and $\varepsilon$ such that for $t \in\left[0, \tau_{0}\right], x_{l}(t) \leqslant x \leqslant x_{r}(t)$, the solution of problem $(2.7),(5.8)-(5.15)$ exists, is Lipschitz continuous, and satisfies

$$
|w(x, t)| \leqslant M_{0}, \quad|\partial w / \partial x|,|\partial w / \partial t| \leqslant M_{1}
$$

Proof. We prove the uniform convergence of the sequence $\left\{w^{(n)}(x, t)\right\}$. Let

$$
\max \left\{\left|u^{(n)}-u^{(n-1)}\right|,\left|p^{(n)}-p^{(n-1)}\right|,\left|c^{(n)}-c^{(n-1)}\right|,\left|s^{(n)}-s^{(n-1)}\right|\right\}=\varepsilon^{(n)} .
$$

We draw a characteristic curve $\xi=\xi(\eta)$ passing through the point $\left(\xi_{1}, \tau_{1}\right)$ on $x=x_{r}(t)$ (see Figure 1) which satisfies

$$
\begin{aligned}
& \frac{d \xi}{d \eta}=\lambda_{4}\left(p^{(n-1)}(\xi, \eta), c^{(n-1)}(\xi, \eta), s^{(n-1)}(\xi, \eta)\right), \\
& \left.\xi\right|_{\eta=\tau_{1}}=\xi_{1} .
\end{aligned}
$$

The intersection point of $\xi=\xi(\eta)$ and the $t$-axis is $(0, \tau)$. Passing $\left(\xi_{1}, \tau_{1}\right)$ we draw another characteristic curve $\xi=\bar{\xi}(\eta)$ which satisfies a similar equation, except exchanging the superscript $n-2$ for $n-1$. The intersection point of $\xi=\bar{\xi}(\eta)$ and 
the $t$-axis is denoted by $(0, \bar{\tau})$. From (5.23),

$$
\begin{aligned}
u^{(n)}+ & \left.l_{42}^{(n-1)} p^{(n)}\right|_{\left(\xi_{1}, \tau_{1}\right)} \\
= & u^{(n)}+\left.l_{42}\left(p^{(n-1)}(0, \tau), c^{(n-1)}(0+, \tau), s^{(n-1)}(0+, \tau)\right) p^{(n)}\right|_{(0, \tau)} \\
& \quad+\int_{\tau}^{\tau_{1}} p^{(n-1)}(\xi(\eta), \eta) \frac{\partial}{\partial \eta} l_{42}\left(p^{(n-1)}(\xi(\eta), \eta), \ldots\right) d \eta, \\
u^{(n-1)}+ & \left.l_{42}^{(n-2)} p^{(n-1)}\right|_{\left(\xi_{1}, \tau_{1}\right)} \\
= & u^{(n-1)}+\left.l_{42}\left(p^{(n-2)}(0, \tau), c^{(n-2)}(0+, \tau), s^{(n-2)}(0+, \tau)\right) p^{(n-1)}\right|_{(0, \bar{\tau})} \\
& +\int_{\bar{\tau}}^{\tau_{1}} p^{(n-2)}(\bar{\xi}(\eta), \eta) \frac{\partial}{\partial \eta} l_{42}\left(p^{(n-2)}(\bar{\xi}(\eta), \eta), \ldots\right) d \eta .
\end{aligned}
$$

Let

$$
\begin{aligned}
& l_{42}^{+}=l_{42}\left(p^{(n-1)}(0, \tau), c^{(n-1)}(0+, \tau), s^{(n-1)}(0+, \tau)\right), \\
& \bar{l}_{42}^{+}=l_{42}\left(p^{(n-2)}(0, \tau), c^{(n-2)}(0+, \tau), s^{(n-2)}(0+, \tau)\right)
\end{aligned}
$$

and subtract the above expressions; we have

$$
\begin{aligned}
\left(u^{(n)}+l_{42}^{(n-1)} p^{(n)}\right)-\left.\left(u^{(n-1)}+l_{42}^{(n-2)} p^{(n-1)}\right)\right|_{\left(\xi_{1}, \tau_{1}\right)} & \left.\left(u^{(n)}+l_{42}^{+} p^{(n)}\right)\right|_{(0, \tau)}-\left.\left(u^{(n-1)}+\bar{l}_{42}^{+} p^{(n-1)}\right)\right|_{(0, \bar{\tau})} \\
& +\int_{\tau}^{\tau_{1}}\left[p^{(n-1)}(\xi(\eta), \eta) \frac{\partial}{\partial \eta} l_{42}\left(p^{(n-1)}(\xi(\eta), \eta), \ldots\right)\right. \\
& \left.-p^{(n-2)}(\bar{\xi}(\eta), \eta) \frac{\partial}{\partial \eta} l_{42}\left(p^{(n-2)}(\bar{\xi}(\eta), \eta), \ldots\right)\right] d \eta \\
& +\int_{\bar{\tau}}^{\tau} p^{(n-2)}(\bar{\xi}(\eta), \eta) \frac{\partial}{\partial \eta} l_{42}\left(p^{(n-2)}(\bar{\xi}(\eta), \eta), \ldots\right) d \eta .
\end{aligned}
$$

From (5.37),

$$
\begin{aligned}
& d(\xi-\bar{\xi}) / d \eta=\left(\partial \lambda_{4} / \partial p\right)\left(p^{(n-1)}(\xi, \eta)-p^{(n-2)}(\bar{\xi}, \eta)\right) \\
&+\left(\partial \lambda_{4} / \partial c\right)\left(c^{(n-1)}(\xi, \eta)-c^{(n-2)}(\bar{\xi}, \eta)\right) \\
&+\left(\partial \lambda_{4} / \partial s\right)\left(s^{(n-1)}(\xi, \eta)-s^{(n-2)}(\bar{\xi}, \eta)\right), \\
& \xi-\left.\bar{\xi}\right|_{\eta=\tau_{1}}=0,
\end{aligned}
$$

where

$$
p^{(n-1)}(\xi, \eta)-p^{(n-2)}(\bar{\xi}, \eta)=\left[p^{(n-1)}(\xi, \eta)-p^{(n-2)}(\xi, \eta)\right]+(\partial p / \partial \xi)(\xi-\bar{\xi}) ;
$$

there are similar expressions for functions $c$ and $s$; hence

$$
|\xi-\bar{\xi}| \leqslant K \varepsilon^{(n-1)} \tau_{0}
$$

It is obvious that

$$
|\tau-\bar{\tau}| \leqslant K \varepsilon^{(n-1)} \tau_{0}
$$

Hence

$$
\left|p^{(n-1)}(\xi(\eta), \eta)-p^{(n-2)}(\bar{\xi}(\eta), \eta)\right|<\varepsilon^{(n-1)}+K \varepsilon^{(n-1)} \tau_{0}
$$


We apply the estimation in Lemma 6 and (5.38)-(5.40) and obtain

$$
\begin{aligned}
\left(u^{(n)}+l_{42}^{(n-1)} p^{(n)}\right)-\left.\left(u^{(n-1)}+l_{42}^{(n-1)} p^{(n-1)}\right)\right|_{\left(\xi_{1}, \tau_{1}\right)} & \\
= & \left(u^{(n)}+l_{42}^{+} p^{(n)}\right)-\left.\left(u^{(n-1)}+l_{42}^{+} p^{(n-1)}\right)\right|_{(0, \tau)}+Q_{1},
\end{aligned}
$$

where $\left|Q_{1}\right| \leqslant K \varepsilon^{(n-1)} \tau_{0}$. Similarly,

$$
\begin{aligned}
\left(u^{(n)}+\right. & \left.l_{42}^{-} p^{(n)}\right)-\left.\left(u^{(n-1)}+l_{42}^{-} p^{(n-1)}\right)\right|_{(0, \tau)} \\
& =\left(u^{(n-1)}+l_{42}^{(n-1)} p^{(n-1)}\right)-\left.\left(u^{(n-2)}+l_{42}^{(n-1)} p^{(n-2)}\right)\right|_{\left(\xi_{2}, \tau_{2}\right)}+Q_{2}, \\
\left(u^{(n)}+\right. & \left.l_{12}^{+} p^{(n)}\right)-\left.\left(u^{(n-1)}+l_{12}^{+} p^{(n-1)}\right)\right|_{(0, \tau)} \\
& =\left(u^{(n-1)}+l_{12}^{(n-1)} p^{(n-1)}\right)-\left.\left(u^{(n-2)}+l_{12}^{(n-1)} p^{(n-2)}\right)\right|_{\left(\xi_{3}, \tau_{3}\right)}+Q_{3},
\end{aligned}
$$

where $Q_{2}, Q_{3}$ have the same estimate as $Q_{1}$. From (5.21) we have

$$
\begin{aligned}
\left(u^{(n)}-\right. & \left.u^{(n-1)}\right)+\left.l_{42}^{(n-1)}\left(p^{(n)}-p^{(n-1)}\right)\right|_{\left(\xi_{1}, \tau_{1}\right)} \\
= & \frac{l_{42}^{+}-l_{12}^{+}}{l_{42}^{-}-l_{12}^{+}}\left[\left(u^{(n-1)}-u^{(n-2)}\right)+l_{42}^{(n-1)}\left(p^{(n-1)}-p^{(n-2)}\right)\right]_{\left(\xi_{2}, \tau_{2}\right)} \\
& +\frac{l_{42}^{-}-l_{42}^{+}}{l_{42}^{-}-l_{12}^{+}}\left[\left(u^{(n-1)}-u^{(n-2)}\right)+l_{12}^{(n-1)}\left(p^{(n-1)}-p^{(n-2)}\right)\right]_{\left(\xi_{3}, \tau_{3}\right)}+Q,
\end{aligned}
$$

where $|Q| \leqslant K \varepsilon^{(n-1)} \tau_{0}$. From (2.13),

$$
\left(u^{(n)}-u^{(n-1)}\right)\left(p^{(n)}-p^{(n-1)}\right)>0 ;
$$

from (2.8),

$$
\frac{\left|\left(u^{(n)}-u^{(n-1)}\right)+l_{12}^{(n-1)}\left(p^{(n)}-p^{(n-1)}\right)\right|}{\left|\left(u^{(n)}-u^{(n-1)}\right)+l_{42}^{n-1)}\left(p^{(n)}-p^{(n-1)}\right)\right|}<\beta<1 .
$$

We note that $u^{(n)}=u^{(n-1)}, p^{(n)}=p^{(n-1)}$ at $\left(\xi_{2}, \tau_{2}\right)$ and $\left|\left(l_{42}^{-}-l_{42}^{+}\right) /\left(l_{42}^{-}-l_{12}^{+}\right)\right|<1$; hence

$$
\begin{gathered}
\left|\left(u^{(n)}-u^{(n-1)}\right)+l_{12}^{(n-1)}\left(p^{(n)}-p^{(n-1)}\right)\right|_{\left(\xi_{1}, \tau_{1}\right)} \\
\leqslant \beta\left|\left(u^{(n-1)}-u^{(n-2)}\right)+l_{12}^{(n-2)}\left(p^{(n-1)}-p^{(n-2)}\right)\right|_{\left(\xi_{3}, \tau_{3}\right)} \\
+\beta\left|l_{12}^{(n-1)}-l_{12}^{(n-2)}\right| \cdot\left|p^{(n-1)}-p^{(n-2)}\right|+Q .
\end{gathered}
$$

Using the boundary conditions (5.9)-(5.11), we can prove that the sequence $\left\{w^{(n)}(x, t)\right\}$ converges uniformly for $\tau_{0}$ small enough. We rewrite (5.23) as

$$
\bar{l}_{i}\left(w^{(n)}\right) \frac{d w^{(n)}}{d t_{i}}=b_{i}\left(w^{(n-1)}\right)+\frac{d \bar{l}_{i}\left(w^{(n-1)}\right)}{d t_{i}}\left(w^{(n-1)}-w^{(n)}\right) .
$$

The term on the right uniformly converges to $b_{i}(u)$; then we obtain the existence of the solution in a similar way to Theorem 2. Q.E.D.

Part 2. Let the slope of the forward shock wave of (3.1), (3.2) be $\lambda$. Then $\lambda<\bar{\lambda}_{4}$. Let $\varepsilon=\left(\bar{\lambda}_{4}-\lambda\right) / 2$ and $\tau_{1} \in\left[0, \tau_{0}\right]$ be a constant to be chosen later, where $\tau_{0}$ is just the constant $\tau_{0}$ defined in Lemma 7. Let $V=\left\{x \mid x \in C^{1}, x(0)=0, x^{\prime}(0)=\lambda\right.$, $\left.\left|x^{\prime}(t)-\lambda\right|<\varepsilon\right\}$ which is a closed convex subset of $C^{1}\left(\left[0, \tau_{1}\right]\right)$.

For any $x \in V$, we take $x(t)$ as $x_{r}(t)$ which occurs in problem (2.7), (5.8)-(5.15). It follows from Lemma 7 that there is a Lipschitz continuous solution $w(x, t)$ such 
that $|w| \leqslant M_{0},|\partial w / \partial x|,|\partial w / \partial t| \leqslant M_{1}$. Solving the Cauchy problem of the system of equations with support $x=x_{r}(t)$ in the direction of decrease in $t$, we obtain the solution, also called $w(x, t)$. There is also a constant $M_{2}$ which depends on $K$ and $\varepsilon$ such that $|w|+|\partial w / \partial x|+|\partial w / \partial t| \leq M_{2}$.

We solve the initial problem of $(2.16)$ using the function $w(x, t)$ that we have just obtained; $y(t)$ denotes the solution. Since the term on the right is Lipschitz continuous and equals $\lambda$ as $t=0$, then $y \in V$ as long as $\tau_{1}$ is assumed such that $\left|y^{\prime}(t)-\lambda\right|<\varepsilon$.

Because $y^{\prime}(t)$ has a uniformly bounded Lipschitz coefficient, i.e. the set $\{y\}$ is bounded in the space $C^{1,1}([0, \tau]), V \rightarrow\{y\}$ is a completely continuous mapping. It follows from the Schauder fixed point theorem that there exists a fixed point in $V$ which is just the shock wave we are looking for. At the same time $w(x, t)$ is also obtained.

6. The other cases of the discontinuous initial problem. For the other cases of the discontinuous initial problem we also can consider the structures of the solution in a way similar to Theorem 3. When the solution of problem (1.6), (1.7) consists of one forward rarefaction wave and one backward shock wave, we can treat the corresponding problem (1.1), (1.5) in the same way as Theorem 3 . When the solution of problem (1.6), (1.7) consists of two rarefaction waves, we also obtain the same result as Theorem 3. In this case we only consider the problem of the rarefaction wave and the fixed boundary problem between the two rarefaction waves.

When the solution of problem (1.6), (1.7) consists of two shock waves, we also can treat the corresponding problem (1.1), (1.5) in a way similar to that in Theorem 3 and obtain a similar result. In this case, although we can obtain (5.32), (5.33) is no longer true; therefore we need the following condition:

$$
\beta\left\{\left|\frac{l_{42}^{+}-l_{12}^{+}}{l_{42}^{-}-l_{12}^{+}}\right|+\left|\frac{l_{42}^{-}-l_{42}^{+}}{l_{42}^{-}-l_{12}^{+}}\right|\right\}<1 .
$$

It is easy to show that when $\left|s_{r}-s_{l}\right|$ and $\left|c_{r}-c_{l}\right|$ are small enough this implies (6.1). So in order to make the structure of the solution clear we must add such kinds of sufficient conditions.

When the solution of problem (1.6)-(1.7) consists of one wave, for example $\bar{p}=p_{r}$ or $\bar{p}=p_{l}$, the structure of problem (1.1), (1.5) at the point 0 cannot be determined by the limits of the initial data at the point 0 . In this case we should consider the derivatives of the initial data at the point 0 .

\section{REFERENCES}

1. F. A. Williams, Combustion theory, Addison-Wesley, Reading, Mass., 1965.

2. K. O. Friedrichs, Nonlinear hyperbolic differential equations for functions of two independent variables, Amer. J. Math. 70 (1948).

3. R. Courant and P. D. Lax, Nonlinear partial differential equations with two independent variables, Comm. Pure Appl. Math. 2 (1949).

4. A. Douglis, Existence theorems for hyperbolic systems, Comm. Pure Appl. Math. 5 (1952).

5. P. Hartman and A. Winter, On hyperbolic partial differential equations, Amer. J. Math. 74 (1952).

6. P. D. Lax, Nonlinear hyperbolic equations, Comm. Pure Appl. Math. 6 (1953). 
7. The initial value problem for nonlinear hyperbolic equations in two independent variables, Ann. of Math. Studies, no. 33, Princeton Univ. Press, Princeton, N. J., 1954.

8. R. Courant and P. D. Lax, Cauchy problem for nonlinear hyperbolic differential equations in two independent variables, Ann. Mat. Pura Appl. (4) 40 (1955).

9. Wang Row-Hwai and Wuu Jwo-Chun, On mixed boundary value problem for quasilinear hyperbolic system of partial differential equation in two independent variables, Acta Sci. Natur. Univ. Kirin 2 (1963), 459-502. (Chinese)

10-12. Gu Chao-Hao, Lee Da-Tsin and Ho Zon-Yi, The Cauchy problem of quasilinear hyperbolic system with disontinuous initial values. I, II, Acta Math. Sinica 11 (1961), 314-329; III, Ibid. 12 (1963), $132-143$.

13. B. Roždestvenskiı̈ and N. Yanenko, The quasilinear systems of equations and their applications to gas dynamics, "Nauka", Moscow, 1968, pp. 538-560.

14-16. Lee Da-Tsin and Yu Wen-Tzu, Some existence theorems for quasilinear hyperbolic systems of partial differential equations in two independent variables. I: Typical boundary value problems, Sci. Sinica 13 (1964), 529-549; II: Typical boundary problems of functional form and typical free boundary problems, Sci. Sinica 13 (1964), 551-562; III: General boundary value problems and general free boundary problems, Sci. Sinica 14 (1965), 1065-1067.

17. , Local solvability for boundary value problems of the quasilinear hyperbolic system, Sci. Sinica and Fudan J. (to appear).

Department of Mathematics, Courant Instiute of Mathematical Sciences, New York UniverSITY, NEW YORK, NEW YORK 10012

Current address (Ying Lung-an): Department of Mathematics, Peking University, Peking, China

Current address (Wang Ching-hua): Institute of Mathematics, Academia Sinica, Peking, China 